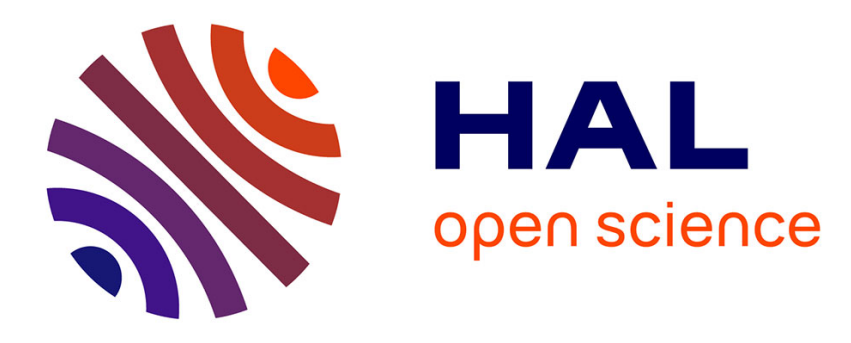

\title{
Unramified -modular representations of GLn(F) and its inner forms
}

\author{
A. Minguez, V. Secherre
}

\section{To cite this version:}

A. Minguez, V. Secherre. Unramified -modular representations of GLn(F) and its inner forms. International Mathematics Research Notices, 2016, 10.1093/imrn/rns278 . hal-01176198

\section{HAL Id: hal-01176198 \\ https://hal.science/hal-01176198}

Submitted on 15 Jul 2015

HAL is a multi-disciplinary open access archive for the deposit and dissemination of scientific research documents, whether they are published or not. The documents may come from teaching and research institutions in France or abroad, or from public or private research centers.
L'archive ouverte pluridisciplinaire HAL, est destinée au dépôt et à la diffusion de documents scientifiques de niveau recherche, publiés ou non, émanant des établissements d'enseignement et de recherche français ou étrangers, des laboratoires publics ou privés. 


\title{
UNRAMIFIED $\ell$-MODULAR REPRESENTATIONS OF GL GL $_{n}(\mathrm{~F})$ AND ITS INNER FORMS
}

\author{
by
}

Alberto Mínguez \& Vincent Sécherre

\begin{abstract}
Let F be a non-Archimedean locally compact field of residue characteristic $p$ and $\mathrm{R}$ be an algebraically closed field of characteristic $\ell$ different from $p$. Let $\mathbf{G}$ be the group $\mathrm{GL}_{n}$, $n \geqslant 1$, over $\mathrm{F}$, or one of its inner forms. In this article, we classify the unramified irreducible smooth R-representations of $\mathbf{G}(\mathrm{F})$ : more precisely we prove that they are those representations that are irreducibly induced from an unramified character of a Levi subgroup. We deduce that any smooth irreducible unramified $\overline{\mathbb{F}}_{\ell}$-representation of $\mathbf{G}(\mathrm{F})$ can be lifted to $\overline{\mathbb{Q}}_{\ell}$, which proves a conjecture by Vignéras.
\end{abstract}

\section{Introduction}

The classification of representations of reductive groups over local fields is a fundamental problem in harmonic analysis, with various applications in the theory of automorphic forms and in number theory. The class of irreducible unramified representations is of particular importance: indeed, if $\mathbf{G}$ is a connected reductive group defined over a number field $k$, and if $\Pi$ is a complex admissible irreducible representation of the adelic group $\mathbf{G}\left(\mathbb{A}_{k}\right)$, then, at almost all finite place $v$, the local component $\Pi_{v}$ is an unramified irreducible complex representation of $\mathbf{G}\left(k_{v}\right)$.

Complex unramified representations of $\mathbf{G}\left(k_{v}\right)$ are well understood (see $[\mathbf{2}, \mathbf{3}, \mathbf{1 0}]$ ). In particular, if $\mathbf{G}=\mathrm{GL}_{n}$, Tadić $[\mathbf{1 1}]$ proved that a complex irreducible representation is unramified if and only if it is irreducibly induced from a unramified character of a Levi subgroup.

So far we have only been concerned with representations on complex vector spaces. Recently however, the applications of the representation theory of $p$-adic reductive groups to number theory have required considering representations with coefficients in fields of positive characteristic (for example, see [4] for the modularity lifting problems). Their behaviour is very different depending on whether this characteristic $\ell$ is equal to, or different from $p$. In this article we will only deal with the latter case. In this case, the theory of $\ell$-modular representations of $p$-adic reductive groups has been developed by Vignéras (see [13]).

This work was partially supported by the ANR (ANR-08-BLAN-0259-01, ANR-10-BLANC-0114) and the EPSRC (EP/G001480/1). First named author was also partially supported by MTM2010-19298 and FEDER. 
Let $\mathrm{F}$ be a non-Archimedean locally compact field of residue characteristic $p$. We write $\mathcal{O}$ for its ring of integers and $q$ for the cardinality of its residue field. Let $\ell \neq p$ be a prime number and $n$ be a positive integer. Irreducible smooth $\ell$-modular representations of the group $\mathrm{GL}_{n}(\mathrm{~F})$ have been studied fifteen years ago [14]. Nevertheless, the classification of those irreducible $\ell-$ modular representations of $\mathrm{GL}_{n}(\mathrm{~F})$ that are unramified, that is, that have nonzero $\mathrm{GL}_{n}(\mathcal{O})$-fixed vectors, remained unknown. Taking $\mathrm{GL}_{n}(\mathcal{O})$-fixed vectors of smooth $\ell$-modular representations of $\mathrm{GL}_{n}(\mathrm{~F})$ is not, in general, an exact functor. There can be more than one irreducible unramified subquotient in an unramified principal series, and this makes things complicated. In particular there might be several unramified $\ell$-modular representations having the same Satake parameter (see $[\mathbf{1 6}$, Ap. B]).

In this article we classify all irreducible unramified $\ell$-modular representations of $\mathrm{GL}_{n}(\mathrm{~F})$ and its inner forms. More precisely, we prove the following theorem.

Theorem 0.1 (see Corollary 6.2). - An irreducible smooth $\ell$-modular representation of the group $\mathrm{GL}_{n}(\mathrm{~F})$ - or one of its inner forms - is unramified if and only if it is irreducibly induced from an unramified character of a Levi subgroup. In this case, the space of its fixed vectors by a maximal compact subgroup has dimension 1. Moreover, any irreducible unramified $\ell$-modular representation can be lifted to an irreducible $\ell$-adic representation.

This theorem was conjectured in [14, VI.3]. To give more detail on the proof let us introduce some notation.

Let $\mathrm{D}$ be a finite dimensional central division F-algebra. For any integer $m \geq 1$, we write $\mathrm{G}_{m}$ for the group $\mathrm{GL}_{m}(\mathrm{D})$. To a cuspidal irreducible $\ell$-modular representation $\rho$ of $\mathrm{G}_{m}$ we attached in $[8]$ an unramified character $\nu_{\rho}$ so that, for any cuspidal irreducible representation $\rho^{\prime}$ of $\mathrm{G}_{m^{\prime}}, m^{\prime} \geq 1$, the (normalized) parabolically induced representation $\rho \times \rho^{\prime}$ is reducible if and only if $m^{\prime}=m$ and $\rho^{\prime}$ is isomorphic to $\rho \nu_{\rho}$ or $\rho \nu_{\rho}^{-1}$. For example, if $\mathrm{D}=\mathrm{F}$, the character $\nu_{\rho}$ is independent of $\rho$ and equals $|\operatorname{det}|_{\mathrm{F}}$, where ||$_{\mathrm{F}}$ denotes the normalized absolute value on $\mathrm{F}$. This allows us to define a segment as a sequence of the form:

$$
[a, b]_{\rho}=\left(\rho \nu_{\rho}^{a}, \rho \nu_{\rho}^{a+1}, \ldots, \rho \nu_{\rho}^{b}\right),
$$

with $a, b \in \mathbb{Z}$ such that $a \leq b$. The support of $[a, b]_{\rho}$ is the multiset (i.e. set with multiplicities) $\rho \nu_{\rho}^{a}+\rho \nu_{\rho}^{a+1}+\cdots+\rho \nu_{\rho}^{b}$, and its length is $b-a+1$. If $a+1 \leqslant b$, we write:

$$
{ }^{-} \Delta=[a+1, b]_{\rho}, \quad \Delta^{-}=[a, b-1]_{\rho} .
$$

To any segment $\Delta=[a, b]_{\rho}$ we have associated in $[\mathbf{9}]$, following Zelevinsky and Vignéras, an irreducible subrepresentation $\mathrm{Z}(\Delta)$ of $\rho \nu_{\rho}^{a} \times \rho \nu_{\rho}^{a+1} \times \cdots \times \rho \nu_{\rho}^{b}$. It is a representation of $\mathrm{G}_{m(b-a+1)}$.

Let $\Delta=[a, b]_{\rho}$ and $\Delta^{\prime}=\left[a^{\prime}, b^{\prime}\right]_{\rho^{\prime}}$ be two segments. We say that $\Delta$ and $\Delta^{\prime}$ are linked if if one can extract from at least one of the following sequences:

$$
\left(\rho \nu_{\rho}^{a}, \ldots, \rho \nu_{\rho}^{b}, \rho^{\prime} \nu_{\rho^{\prime}}^{a^{\prime}}, \ldots, \rho^{\prime} \nu_{\rho^{\prime}}^{b^{\prime}}\right) \text { and }\left(\rho^{\prime} \nu_{\rho^{\prime}}^{a^{\prime}}, \ldots, \rho^{\prime} \nu_{\rho^{\prime}}^{b^{\prime}}, \rho \nu_{\rho}^{a}, \ldots, \rho \nu_{\rho}^{b}\right)
$$

a subsequence which is a segment of length greater than those of $\Delta$ and $\Delta^{\prime}$.

If $\Delta_{1}, \ldots, \Delta_{r}$ are segments we know that the induced representation $\mathrm{Z}\left(\Delta_{1}\right) \times \cdots \times \mathrm{Z}\left(\Delta_{r}\right)$ is irreducible if and only if, for all $i, j \in\{1, \ldots, r\}, i \neq j$, the segments $\Delta_{i}$ and $\Delta_{j}$ are not linked. 
We deduce in section 3 that, if $\Delta_{1}, \ldots, \Delta_{r}$ are segments with support made of unramified characters and for all $i, j \in\{1, \ldots, r\}, i \neq j$, the segments $\Delta_{i}$ and $\Delta_{j}$ are not linked, then the representation $\mathrm{Z}\left(\Delta_{1}\right) \times \cdots \times \mathrm{Z}\left(\Delta_{r}\right)$ is irreducible and unramified. The hard task is to prove that every irreducible unramified $\ell$-modular representation is of that form.

It is easy to see, by the Iwasawa decomposition, that such a representation is a subquotient of a principal unramified series. These representations are parametrized by unramified multisegments, that is multisets of segments with support made of unramified characters. Let $e$ be the multiplicative order of the cardinality of the residue field of $\mathrm{D}$ modulo $\ell$. To prove Theorem 0.1 we distinguish two cases, depending on whether $e=1$ or not.

The case when $e=1$ is easy, and the proof was already sketched in [14, VI.2]. It relies on the use of the Hecke-Iwahori algebra. See Section 4 for more details.

The case when $e>1$ is more complicated. The key idea is that, in this case, on can distinguish irreducible $\ell$-modular representations by their proper Jacquet modules. By an inductive argument carried out in Section 6, one can see that it is enough to prove that, if $\Delta=[a, b]_{\rho}$ and $\Delta^{\prime}=\left[a^{\prime}, b^{\prime}\right]_{\rho}$ are two linked segments, with $\rho$ an unramified character of $\mathrm{G}_{1}$, then the irreducible representation corresponding to the multisegment $\Delta+\Delta^{\prime}$, denoted $\mathrm{Z}\left(\Delta+\Delta^{\prime}\right)$, is not unramified. We prove this result in Section 5. For this we distinguish several cases and see that $\mathrm{Z}\left(\Delta+\Delta^{\prime}\right)$ is always a subrepresentation of one of the following induced representations:

(1) $\mathrm{Z}\left(\Delta^{-}+\Delta^{\prime-}\right) \times \rho \nu_{\rho}^{b} \times \rho \nu_{\rho}^{b^{\prime}}$, with $\Delta^{-}, \Delta^{\prime-}$ linked;

(2) $\mathrm{Z}\left(\Delta+\Delta^{\prime-}\right) \times \rho \nu_{\rho}^{b^{\prime}}$, with $\Delta, \Delta^{\prime-}$ linked;

(3) $\rho \nu_{\rho}^{a} \times \rho \nu_{\rho}^{a^{\prime}} \times \mathrm{Z}\left({ }^{-} \Delta+{ }^{-} \Delta^{\prime}\right)$, with ${ }^{-} \Delta,{ }^{-} \Delta^{\prime}$ linked;

(4) $\rho \nu_{\rho}^{a^{\prime}} \times \mathrm{Z}\left(\Delta+{ }^{-} \Delta^{\prime}\right)$, with $\Delta,{ }^{-} \Delta^{\prime}$ linked;

and the theorem follows from an inductive argument. See Section 5 for more details.

\section{Acknowledgements}

The authors would like to thank Shaun Stevens for useful discussions.

\section{Notation and preliminaries}

1.1. Throughout this article, $\mathrm{F}$ is a non-Archimedean locally compact field of residue characteristic $p$ and $q$ is the cardinality of its residue field. Let $\mathrm{D}$ be a finite dimensional central division F-algebra of reduced degree $d$ and let $\mathcal{O}_{\mathrm{D}}$ be its ring of integers. Let $\mathrm{R}$ be an algebraically closed field of characteristic $\ell$ different from $p$. If $\ell>0$, we denote by $e$ the order of $q^{d}$ in $\mathrm{R}^{\times}$. If $\ell=0$, we set $e=\infty$.

1.2. A smooth $\mathrm{R}$-representation of a topological group $\mathrm{G}$ is a pair $(\pi, \mathrm{V})$ where $\mathrm{V}$ is a $\mathrm{R}$-vector space and $\pi$ is a group homomorphism from $G$ to $\operatorname{Aut}_{\mathrm{R}}(\mathrm{V})$ such that the stabilizer of any vector in $\mathrm{V}$ is an open subgroup in $\mathrm{G}$. In this article all representations will be smooth.

An $\mathrm{R}$-character of $\mathrm{G}$ is a group homomorphism from $\mathrm{G}$ to $\mathrm{R}^{\times}$with open kernel. It is a smooth representation of dimension 1. Given $\pi$ an R-representation and $\chi$ an R-character of $\mathrm{G}$, we write $\chi \pi$ or $\pi \chi$ for the representation $g \mapsto \chi(g) \pi(g)$. 
We denote by $\operatorname{Irr}_{\mathrm{R}}(\mathrm{G})$ the set of equivalence classes of irreducible R-representations of G. For any finite length $\mathrm{R}$-representation $\pi$ of $\mathrm{G}$ we will denote by $[\pi]$ its semisimplification.

When the coefficient field is clear from the context, we will write character and representation rather than R-character and R-representation.

1.3. For any $m \geq 1$, we write $\mathscr{M}_{m}(\mathrm{D})$ for the F-algebra of $m \times m$ matrices with coefficients in $\mathrm{D}$ and $\mathrm{G}_{m}=\mathrm{GL}_{m}(\mathrm{D})$ for the group of its invertible elements.

Let $\mathrm{N}_{m}$ denote the reduced norm from $\mathscr{M}_{m}(\mathrm{D})$ to $\mathrm{F}$, and write ||$_{\mathrm{F}}$ for the normalized absolute value of $\mathrm{F}$, giving the value $q^{-1}$ to any uniformizer of $\mathrm{F}$. As the image of $q$ in $\mathrm{R}$ is invertible, this defines an R-character of $\mathrm{F}^{\times}$that we will write ||$_{\mathrm{F}, \mathrm{R}}$. The map $g \mapsto\left|\mathrm{N}_{m}\right|_{\mathrm{F}, \mathrm{R}}$ is an R-character of $\mathrm{G}_{m}$ that we denote by $\nu$ (the $m$ will be implicit and hopefuly clear from the context).

If $\pi$ is an R-representation of $\mathrm{G}_{m}$ we will write $\operatorname{deg}(\pi)=m$, the degree of $\pi$.

1.4. Let $m$ be a positive integer. A composition of $m$ is a finite sequence $\alpha=\left(m_{1}, \ldots, m_{r}\right)$ of positive integers of sum $m$. If $m_{i} \geq m_{i+1}$ for all $1 \leq i \leq r-1$, we say that $\alpha$ is a partition of $m$. To each composition $\alpha=\left(m_{1}, \ldots, m_{r}\right)$ of $m$ we naturally associate a unique partition by rearranging the $m_{i}$ 's. We define a partial order in the set of partitions of the integer $m$. If $\alpha=\left(n_{1}, \ldots, n_{s}\right)$ and $\beta=\left(m_{1}, \ldots, m_{r}\right)$ are two partitions of $m$, we write $\alpha \unlhd \beta$ if:

$$
n_{1}+\cdots+n_{k} \leqslant m_{1}+\cdots+m_{k}
$$

for all integers $k \geqslant 1$. We write $\alpha \triangleleft \beta$ if we have in addition $\alpha \neq \beta$.

Lemma 1.1. - Let $\alpha=\left(n_{1}, \ldots, n_{r}\right)$ and $\beta=\left(m_{1}, \ldots, m_{r}\right)$ be two partitions of $m$. Assume there are two integers $1 \leq i<j \leq r$ such that:

(1) $n_{k}=m_{k}$ if $k \notin\{i, j\}$.

(2) $n_{i}>m_{i}$.

Then we have $\alpha \triangleright \beta$.

Proof. - See that $n_{j}=m_{j}+m_{i}-n_{i}$. By definition of the order we can suppose first $i=1$ and $j=r$. Then we can suppose $n_{j}=0$. The proof is now straightforward.

1.5. Let $\alpha=\left(m_{1}, \ldots, m_{r}\right)$ be a composition of $m$. We denote by $\mathrm{M}_{\alpha}$ the subgroup of $\mathrm{G}_{m}$ of invertible matrices which are diagonal by blocks of size $m_{1}, \ldots, m_{r}$ respectively (it is isomorphic to $\mathrm{G}_{m_{1}} \times \cdots \times \mathrm{G}_{m_{r}}$ ) and by $\mathrm{P}_{\alpha}$ the subgroup of $\mathrm{G}_{m}$ generated by $\mathrm{M}_{\alpha}$ and the upper triangular matrices. A standard parabolic subgroup of $\mathrm{G}_{m}$ is a subgroup of the form $\mathrm{P}_{\alpha}$ and its Levi factor is $\mathrm{M}_{\alpha}$.

We choose once and for all a square root of $q$ in $\mathrm{R}$. Write $\boldsymbol{r}_{\alpha}$ for the normalized Jacquet functor associated to $\mathrm{P}_{\alpha}$ and $\boldsymbol{i}_{\alpha}$ for its right adjoint functor, that is, normalized parabolic induction. If $\pi_{1}, \ldots, \pi_{r}$ are smooth representations of $\mathrm{G}_{m_{1}}, \ldots, \mathrm{G}_{m_{r}}$ respectively, we write:

$$
\pi_{1} \times \pi_{2} \times \cdots \times \pi_{r}=\boldsymbol{i}_{\alpha}\left(\pi_{1} \otimes \pi_{2} \otimes \cdots \otimes \pi_{r}\right) .
$$


1.6. In this paragraph we give a combinatorial version of the Geometric Lemma of BernsteinZelevinsky [1] (see also [13, II.2.19]). Let $\alpha=\left(m_{1}, \ldots, m_{r}\right)$ and $\beta=\left(n_{1}, \ldots, n_{s}\right)$ be two compositions of an integer $m \geqslant 1$. For every $i \in\{1, \ldots, r\}$, let $\pi_{i}$ be an irreducible R-representation of $\mathrm{G}_{m_{i}}$, and let $\pi=\pi_{1} \otimes \cdots \otimes \pi_{r} \in \operatorname{Irr}_{\mathrm{R}}\left(\mathrm{M}_{\alpha}\right)$. Denote by $\mathscr{M}^{\alpha, \beta}$ the set of matrices $\mathrm{B}=\left(b_{i, j}\right)$ with non-negative coefficients such that:

$$
\sum_{j=1}^{s} b_{i, j}=m_{i}, \quad i \in\{1, \ldots, r\}, \quad \sum_{i=1}^{r} b_{i, j}=n_{j}, \quad j \in\{1, \ldots, s\} .
$$

Fix $\mathrm{B} \in \mathscr{M}^{\alpha, \beta}$ and denote by $\alpha_{i}=\left(b_{i, 1}, \ldots, b_{i, s}\right)$ and $\beta_{j}=\left(b_{1, j}, \ldots, b_{r, j}\right)$ which are compositions of $m_{i}$ and $n_{j}$ respectively. For all $i \in\{1, \ldots, r\}$, set:

$$
\sigma_{i}^{(k)}=\sigma_{i, 1}^{(k)} \otimes \cdots \otimes \sigma_{i, s}^{(k)}, \quad \sigma_{i, j}^{(k)} \in \operatorname{Irr}\left(\mathrm{G}_{b_{i, j}}\right), \quad k \in\left\{1, \ldots, r_{i}\right\},
$$

the different composition factors of $\boldsymbol{r}_{\alpha_{i}}\left(\pi_{i}\right)$. For all $j \in\{1, \ldots, s\}$ and all sequences of integers $\left(k_{1}, \ldots, k_{r}\right)$ such that $1 \leqslant k_{i} \leqslant r_{i}$, we define the representation $\sigma_{j}$ of $\mathrm{G}_{n_{j}}$ by:

$$
\sigma_{j}=\boldsymbol{i}_{\beta_{j}}\left(\sigma_{1, j}^{\left(k_{1}\right)} \otimes \cdots \otimes \sigma_{r, j}^{\left(k_{r}\right)}\right) .
$$

Then we have:

$$
\left[\boldsymbol{r}_{\beta} \circ \boldsymbol{i}_{\alpha}(\pi)\right]=\sum_{\mathrm{B} \in \mathscr{M}^{\alpha, \beta},\left(k_{1}, \ldots, k_{r}\right)}\left[\sigma_{1} \otimes \cdots \otimes \sigma_{s}\right]
$$

1.7. An irreducible representation $\pi$ of $\mathrm{G}_{m}$ is said to be cuspidal if it is not a subrepresentation (or equivalently a quotient) of an induced representation of the form (1.1) with $r \geq 2$ and $\pi_{1}, \ldots, \pi_{r}$ all irreducible. It is said to be supercuspidal if it is not a subquotient of a representation of the form (1.1) with $r \geq 2$ and $\pi_{1}, \ldots, \pi_{r}$ irreducible. A supercuspidal irreducible representation is always cuspidal but, in general, the converse is not true. Write $\mathcal{C}_{\mathrm{R}}\left(\mathrm{G}_{m}\right)$ and $\mathcal{S}_{\mathrm{R}}\left(\mathrm{G}_{m}\right)$ for the subsets of $\operatorname{Irr}_{\mathrm{R}}\left(\mathrm{G}_{m}\right)$ made of equivalence classes of cuspidal and supercuspidal representations of $\mathrm{G}_{m}$. We write $\mathcal{C}_{\mathrm{R}}$ and $\mathcal{S}_{\mathrm{R}}$ for the disjoint union of $\mathcal{C}_{\mathrm{R}}\left(\mathrm{G}_{m}\right)$ and $\mathcal{S}_{\mathrm{R}}\left(\mathrm{G}_{m}\right)$ respectively, for $m \geqslant 1$.

Given a set $\mathrm{X}$, write $\mathbb{N}(\mathrm{X})$ for the commutative semigroup of maps from $\mathrm{X}$ to $\mathbb{N}$ with finite support, and $\leqslant$ for the natural partial order on $\mathbb{N}(X)$.

For any irreducible representation $\pi$ of $\mathrm{G}_{m}$ there exists a composition $\alpha=\left(m_{1}, \ldots, m_{r}\right)$ of $m$ and cuspidal (resp. supercuspidal) representations $\pi_{1}, \ldots, \pi_{r}$ of $\mathrm{G}_{m_{1}}, \ldots, \mathrm{G}_{m_{r}}$ such that $\pi$ is a subrepresentation (resp. a subquotient) of $\pi_{1} \times \pi_{2} \times \cdots \times \pi_{r}$. The sum:

$$
\left[\pi_{1}\right]+\left[\pi_{2}\right]+\cdots+\left[\pi_{r}\right]
$$

in $\mathbb{N}\left(\mathcal{C}_{\mathrm{R}}\right)$ (resp. in $\mathbb{N}\left(\mathcal{S}_{\mathrm{R}}\right)$ ) is unique and called the cuspidal (resp. supercuspidal) support of $\pi$ (see [9, Théorèmes 2.1 et 8.16$]$ ).

1.8. We write:

$$
\mathrm{K}_{0}=\mathrm{GL}_{m}\left(\mathcal{O}_{\mathrm{D}}\right)
$$

for the subgroup of $\mathrm{G}_{m}$ made of those elements $g \in \mathscr{M}_{m}\left(\mathcal{O}_{\mathrm{D}}\right)$ such that $\mathrm{N}_{m}(g)$ is a unit in $\mathcal{O}_{\mathrm{D}}^{\times}$. Any maximal compact subgroup of $\mathrm{G}_{m}$ is conjugate to $\mathrm{K}_{0}$, and any such subgroup is open. 
An R-representation of $\mathrm{G}_{m}$ is said to be unramified (or spherical) if it has a non-zero K-fixed vector for a maximal compact subgroup $\mathrm{K}_{\text {of }} \mathrm{G}_{m}$. The notion of unramified representation does not depend on the choice of $\mathrm{K}$ because all maximal compact subgroups are conjugated in $\mathrm{G}_{m}$.

An R-character of $\mathrm{G}_{1}=\mathrm{D}^{\times}$is unramified if and only if it is trivial on the unit subgroup $\mathcal{O}_{\mathrm{D}}^{\times}$. Every unramified character of $\mathrm{G}_{1}$ is of the form $\chi \circ \mathrm{N}_{1}$ with $\chi$ an unramified character of $\mathrm{F}^{\times}$(it is determined by the image of any uniformizer of $\mathrm{D}$ ).

\section{On the classification of irreducible R-representations of $G_{m}$}

In this section, we recall the classification of irreducible representations of $\mathrm{G}_{m}$, with $m \geqslant 1$, in terms of multisegments (see $[\mathbf{9}]$ ).

2.1. Let $m \geq 1$ be an integer and $\rho$ be an irreducible cuspidal R-representation of $\mathrm{G}_{m}$. In $[8]$ we associate to $\rho$ an unramified character $\nu_{\rho}$ of $\mathrm{G}_{m}$ such that, if $\rho^{\prime}$ is an irreducible cuspidal representation of $\mathrm{G}_{m^{\prime}}$ for some $m^{\prime} \geq 1$, the induced representation $\rho \times \rho^{\prime}$ is reducible if and only if $m^{\prime}=m$ and $\rho^{\prime}$ is isomorphic to $\rho \nu_{\rho}$ or $\rho \nu_{\rho}^{-1}$. We set:

$$
\mathbb{Z}_{\rho}=\left\{\left[\rho \nu_{\rho}^{i}\right] \mid i \in \mathbb{Z}\right\} .
$$

In the case where the characteristic of $\mathrm{R}$ is non-zero, this set is finite and we denote by $e(\rho)$ its cardinality. If the characteristic of $\mathrm{R}$ is zero, $\mathbb{Z}_{\rho}$ is an infinite set and we write $e(\rho)=\infty$.

Example 2.1. - If $\rho$ is the trivial representation of $\mathrm{G}_{1}$, or more generally, if $\rho$ is a character of $\mathrm{G}_{1}$ then $e(\rho)=e$ (see Paragraph 1.1) and $\nu_{\rho}=\nu^{d}$.

Definition 2.2. - A segment is a finite sequence of the form:

$$
\left(\rho \nu_{\rho}^{a}, \rho \nu_{\rho}^{a+1}, \ldots, \rho \nu_{\rho}^{b}\right)
$$

where $\rho$ is as above and $a, b \in \mathbb{Z}$ are two integers such that $a \leqslant b$. Such a sequence is denoted $[a, b]_{\rho}$.

If $\Delta=[a, b]_{\rho}$ is a segment, we write:

$$
l(\Delta)=b-a+1, \quad \operatorname{deg}(\Delta)=(b-a+1) m,
$$

respectively the length and the degree of $\Delta$. If $a+1 \leqslant b$, we set:

$$
{ }^{-} \Delta=[a+1, b]_{\rho}, \quad \Delta^{-}=[a, b-1]_{\rho} .
$$

The support of $\Delta$, denoted $\operatorname{supp}(\Delta)$, is the sum:

$$
\operatorname{supp}(\Delta)=\left[\rho \nu_{\rho}^{a}\right]+\cdots+\left[\rho \nu_{\rho}^{b}\right]
$$

in $\mathbb{N}\left(\mathfrak{C}_{\mathrm{R}}\right)$.

Definition 2.3. - Let $\Delta=[a, b]_{\rho}$ and $\Delta^{\prime}=\left[a^{\prime}, b^{\prime}\right]_{\rho^{\prime}}$ be two segments.

(1) We say that $\Delta$ precedes $\Delta^{\prime}$ if one can extract from the sequence:

$$
\left(\rho \nu_{\rho}^{a}, \ldots, \rho \nu_{\rho}^{b}, \rho^{\prime} \nu_{\rho^{\prime}}^{a^{\prime}}, \ldots, \rho^{\prime} \nu_{\rho^{\prime}}^{b^{\prime}}\right)
$$

a subsequence which is a segment of length greater than $l(\Delta)$ and $l\left(\Delta^{\prime}\right)$. 
(2) We say that $\Delta$ and $\Delta^{\prime}$ are linked if $\Delta$ precedes $\Delta^{\prime}$ or if $\Delta^{\prime}$ precedes $\Delta$.

(3) We say that $\Delta$ and $\Delta^{\prime}$ are equivalent if they have the same length and $\rho \nu_{\rho}^{a}$ is isomorphic to $\rho^{\prime} \nu_{\rho^{\prime}}^{a^{\prime}}$.

Remark that, if $\Delta$ and $\Delta^{\prime}$ are linked or equivalent, then $\mathbb{Z}_{\rho}=\mathbb{Z}_{\rho^{\prime}}$. Conversely, when $\mathbb{Z}_{\rho}=\mathbb{Z}_{\rho^{\prime}}$, we have the following results.

Lemma 2.4. - Let $\Delta=[a, b]_{\rho}$ and $\Delta^{\prime}=\left[a^{\prime}, b^{\prime}\right]_{\rho}$ be two segments. Then $\Delta$ precedes $\Delta^{\prime}$ if and only if the two following conditions are fulfilled:

(1) There is a $c^{\prime} \in \mathbb{Z}$ such that $a^{\prime} \leqslant c^{\prime} \leqslant b^{\prime}$ and $c^{\prime} \equiv b+1(\bmod e(\rho))$.

(2) There is $a c \in \mathbb{Z}$ such that $a \leqslant c \leqslant b$ and $c \equiv a^{\prime}-1(\bmod e(\rho))$.

Remark 2.5. - Note that Condition 1 is enough when $l(\Delta) \geqslant l\left(\Delta^{\prime}\right)$, and Condition 2 is enough when $l(\Delta) \leqslant l\left(\Delta^{\prime}\right)$.

Proof. - By Remark 2.5, the two conditions imply that $\Delta$ precedes $\Delta^{\prime}$. Assume that $\Delta$ precedes $\Delta^{\prime}$. There are integers $a \leqslant c_{1}<c_{2}<\cdots<c_{s} \leqslant b$ and $a^{\prime} \leqslant c_{s+1}<c_{s+2}<\cdots<c_{r} \leqslant b^{\prime}$ such that:

(1) one has $c_{i+1} \equiv c_{i}+1(\bmod e(\rho))$ for all $i \in\{1, \ldots, r-1\}$;

(2) the integer $r$ is greater than $l(\Delta)$ and $l\left(\Delta^{\prime}\right)$.

By adding the integers that may be missing, we may assume that $\left\{c_{1}, \ldots, c_{s}\right\}=\left\{a, a+1, \ldots, c_{s}\right\}$ and that $\left\{c_{s+1}, \ldots, c_{r}\right\}=\left\{c_{s+1}, \ldots, b^{\prime}-1, b^{\prime}\right\}$. Thus we have $c_{s}=a+s-1$ and $c_{s+1}=b^{\prime}+1-r+s$. Since $l(\Delta)<r$, we get $b-c_{s}<r-s$. Then $c^{\prime}=c_{s+1}+b-c_{s}$ satisfies Condition 1. Similarly, since $l\left(\Delta^{\prime}\right)<r$, we get $c_{s+1}-a^{\prime}<s$. Then $c=c_{s}+a^{\prime}-c_{s+1}$ satisfies Condition 2 .

We deduce the following corollary.

Corollary 2.6. - Let $\Delta=[a, b]_{\rho}$ and $\Delta^{\prime}=\left[a^{\prime}, b^{\prime}\right]_{\rho}$ be two segments such that $l(\Delta) \geqslant l\left(\Delta^{\prime}\right)$. Then $\Delta$ and $\Delta^{\prime}$ are linked if and only if there is a $c^{\prime} \in \mathbb{Z}$ such that $a^{\prime} \leqslant c^{\prime} \leqslant b^{\prime}$ and $c^{\prime}$ is congruent to $b+1$ or $a-1 \bmod e(\rho)$.

We write $\mathrm{Seg}=\mathrm{Seg}_{\mathrm{R}}$ for the set of equivalence classes of segments.

Definition 2.7. - A multisegment is a multiset of equivalence classes of segments, that is an element in $\mathbb{N}(\mathrm{Seg})$. We write Mult $=$ Mult $_{\mathrm{R}}$ for the set of multisegments.

Let $\mathfrak{m}=\Delta_{1}+\cdots+\Delta_{r}$ be a multisegment. The length, degree and support can be extended additively to Mult, that is we will write:

$$
l(\mathfrak{m})=\sum_{1 \leqslant i \leqslant r} l\left(\Delta_{i}\right), \quad \operatorname{deg}(\mathfrak{m})=\sum_{1 \leqslant i \leqslant r} \operatorname{deg}\left(\Delta_{i}\right), \quad \operatorname{supp}(\mathfrak{m})=\sum_{1 \leqslant i \leqslant r} \operatorname{supp}\left(\Delta_{i}\right),
$$

the length, the degree and the support of $\mathfrak{m}$ respectively.

Definition 2.8. - A segment $\Delta=[a, b]_{\rho}$ is said to be supercuspidal if $\rho$ is supercuspidal, and unramified if $\rho$ is an unramified character of $\mathrm{G}_{1}$.

We say that $\mathfrak{m}$ is supercuspidal if $\Delta_{i}$ is supercuspidal for all $1 \leq i \leq r$, and that $\mathfrak{m}$ is unramified if $\Delta_{i}$ is unramified for all $1 \leq i \leq r$. 
2.2. Let $m \geq 1$ be a positive integer and $\rho$ be a cuspidal representation of $\mathrm{G}_{m}$. To any segment $\Delta=[a, b]_{\rho}$ of degree $n$, we associate in $[\mathbf{9}, \S 7.2]$ a representation:

\section{$\mathrm{Z}(\Delta)$}

of $\mathrm{G}_{n}$ which is a subrepresentation of $\rho \nu_{\rho}^{a} \times \rho \nu_{\rho}^{a+1} \times \cdots \times \rho \nu_{\rho}^{b}$. This representation satisfies the property:

$$
\boldsymbol{r}_{(m, n-m)}(\mathrm{Z}(\Delta))=\rho \nu_{\rho}^{a} \otimes \mathrm{Z}\left({ }^{-} \Delta\right)
$$

and, if $e(\rho) \geq 2$, this property characterises $\mathrm{Z}(\Delta)$ inductively. See $[\mathbf{9}, \S 7.2]$ for more details.

By a slight abuse of notation, we will consider the case where $b$ is equal to $a-1$ and associate to $[a, a-1]_{\rho}$ the one-dimensional representation of the trivial group, denoted $\mathrm{G}_{0}$.

Example 2.9. - Suppose that $\rho$ is the trivial representation of $\mathrm{G}_{1}$, denoted 1. Then $\mathrm{Z}\left([a, b]_{1}\right)$ is the unramified character $\nu^{(a+b) / 2}$ of $\mathrm{G}_{b-a+1}$. By $[\mathbf{9}, \S 7.2]$, if $\chi$ is a character of $\mathrm{F}^{\times}$, we have:

$$
\mathrm{Z}\left([a, b]_{\chi \circ \mathrm{N}_{1}}\right)=\left(\chi \circ \mathrm{N}_{b-a+1}\right) \mathrm{Z}\left([a, b]_{1}\right) \text {. }
$$

In particular, if $\mu$ is a character of $\mathrm{G}_{1}$, the representation $\mathrm{Z}\left([a, b]_{\mu}\right)$ is a character of $\mathrm{G}_{b-a+1}$.

One of the main ingredients we will use in this article is the following theorem.

Theorem 2.10 (see Théorème $\mathbf{7 . 2 3}$ in [9]). - Let $r \geqslant 1$ be an integer and let $\Delta_{1}, \ldots, \Delta_{r}$ be segments. The following statements are equivalent:

(1) For all $i, j \in\{1, \ldots, r\}, i \neq j$, the segments $\Delta_{i}$ and $\Delta_{j}$ are not linked.

(2) The induced representation $\mathrm{Z}\left(\Delta_{1}\right) \times \cdots \times \mathrm{Z}\left(\Delta_{r}\right)$ is irreducible.

If one of these conditions is fulfilled, then for any permutation $\sigma \in \mathcal{S}_{r}$, the irreducibly induced representations $\mathrm{Z}\left(\Delta_{1}\right) \times \cdots \times \mathrm{Z}\left(\Delta_{r}\right)$ and $\mathrm{Z}\left(\Delta_{\sigma(1)}\right) \times \cdots \times \mathrm{Z}\left(\Delta_{\sigma(r)}\right)$ are isomorphic.

We classified in [9] the irreducible representations of $\mathrm{G}_{n}$, for all $n \geqslant 1$, in terms of multisegments. More precisely, we defined a map:

$$
\mathfrak{m} \mapsto \mathrm{Z}(\mathfrak{m})
$$

that associates to any multisegment $\mathfrak{m}=\Delta_{1}+\cdots+\Delta_{r}$ an irreducible subquotient $\mathrm{Z}(\mathfrak{m})$ of the induced representation:

$$
\mathrm{Z}\left(\Delta_{1}\right) \times \cdots \times \mathrm{Z}\left(\Delta_{r}\right)
$$

that satisfies one condition that will not be very important to us in this article. See $[\mathbf{9}, \S 9]$ for more details; we will explicit the construction in the case where $r=2$ in the next paragraph. This map induces a bijection between supercuspidal multisegments of degree $n$ and irreducible representations of $\mathrm{G}_{n}$.

2.3. We need to give more details about the construction of $Z(\mathfrak{m})$ from the multisegment $\mathfrak{m}$. To do so, we introduce the notion of residually nondegenerate representation $[\mathbf{9}, \S 8]$. We will need the definition only in the case of representations with cuspidal support of cardinality at most two, a very simple case. Then we will explain how to define $\mathrm{Z}(\mathfrak{m})$ when the multisegment $\mathfrak{m}$ has exactly two segments. 
2.3.1. The concept of residually non-degenerate representation generalizes to inner forms of $\mathrm{GL}_{n}(\mathrm{~F})$ the notion of non-degenerate representation (developed in $[\mathbf{1 3}]$ for R-representations). In particular a cuspidal irreducible representation is always residually non-degenerate.

Let $m, m^{\prime}$ be two positive integers and let $\rho$ and $\rho^{\prime}$ be respectively two cuspidal representations of $\mathrm{G}_{m}$ and $\mathrm{G}_{m^{\prime}}$. We define an irreducible representation $\operatorname{St}\left(\rho, \rho^{\prime}\right)$ of $\mathrm{G}_{m+m^{\prime}}$ as follows:

(1) If $\rho^{\prime}$ is not isomorphic to $\rho \nu_{\rho}$ nor to $\rho \nu_{\rho}^{-1}$ (see Paragraph 2.1), the induced representation $\rho \times \rho^{\prime}$ is irreducible, and we set $\operatorname{St}\left(\rho, \rho^{\prime}\right)=\rho \times \rho^{\prime}$.

(2) If $\rho^{\prime}$ is isomorphic to $\rho \nu_{\rho}$, then $\mathrm{Z}\left([0,1]_{\rho}\right)$ is a submodule of $\rho \times \rho^{\prime}$ and:

- if $e(\rho) \geq 3$, then $\rho \times \rho^{\prime}$ has length 2 , and we write $\operatorname{St}\left(\rho, \rho^{\prime}\right)$ for its unique irreducible quotient;

- if $e(\rho)=2$ or if $\ell=2$, then $\rho \times \rho^{\prime}$ has length 3 , with $\mathrm{Z}\left([1,2]_{\rho}\right)$ as a unique quotient, and with a cuspidal irreducible subquotient, which we write $\operatorname{St}\left(\rho, \rho^{\prime}\right)$;

- if $e(\rho)=1$ and $\ell \neq 2$, then $\rho \times \rho^{\prime}$ is a semisimple representation of length 2: we write $\operatorname{St}\left(\rho, \rho^{\prime}\right)$ for the unique irreducible subquotient which is not isomorphic to $\mathrm{Z}\left([0,1]_{\rho}\right)$.

In all cases we have defined an irreducible representation $\operatorname{St}\left(\rho, \rho^{\prime}\right)$ : this is the unique irreducible residually non-degenerate subquotient of $\rho \times \rho^{\prime}$ in the sense of $[\mathbf{9}]$.

If $\alpha=\left(m_{1}, \ldots, m_{r}\right)$ is a composition of $m$, a residually non-degenerate representation of the Levi subgroup $M_{\alpha}$ of $G_{m}$ is a tensor product of residually non-degenerate representations of the various $\mathrm{G}_{m_{i}}$ 's.

2.3.2. Let $m$ be a positive integer and let $\rho$ be a cuspidal representation of $\mathrm{G}_{m}$. Let $a, b, a^{\prime}, b^{\prime}$ some integers such that $a \leq b$ and $a^{\prime} \leq b^{\prime}$. Write $\Delta=[a, b]_{\rho}$ and $\Delta^{\prime}=\left[a^{\prime}, b^{\prime}\right]_{\rho}$. Let $l$ and $l^{\prime}$ be the lengths of $\Delta$ and $\Delta^{\prime}$ respectively and suppose that $l \geq l^{\prime}$. We define two compositions:

$$
\begin{aligned}
& \alpha_{\Delta, \Delta^{\prime}}^{<}=(\underbrace{m, m, \ldots, m}_{l-l^{\prime} \text { times }}, \underbrace{2 m, 2 m, \ldots, 2 m}_{l^{\prime} \text { times }}), \\
& \alpha_{\Delta, \Delta^{\prime}}^{>}=(\underbrace{2 m, 2 m, \ldots, 2 m}_{l^{\prime} \text { times }}, \underbrace{m, m, \ldots, m}_{l-l^{\prime} \text { times }})
\end{aligned}
$$

of $\left(l+l^{\prime}\right) m$ and write $\tau_{\Delta, \Delta^{\prime}}^{<}$and $\tau_{\Delta, \Delta^{\prime}}^{>}$for the representations of $\mathrm{M}_{\alpha_{\Delta, \Delta^{\prime}}^{<}}$and $\mathrm{M}_{\alpha_{\Delta, \Delta^{\prime}}}$, defined by:

$$
\begin{aligned}
\tau_{\Delta, \Delta^{\prime}}^{<} & =\rho \nu_{\rho}^{a} \otimes \rho \nu_{\rho}^{a+1} \otimes \cdots \otimes \rho \nu_{\rho}^{b+b^{\prime}-a^{\prime}-1} \otimes \operatorname{St}\left(\rho \nu_{\rho}^{b+b^{\prime}-a^{\prime}}, \rho \nu_{\rho}^{a^{\prime}}\right) \otimes \cdots \otimes \operatorname{St}\left(\rho \nu_{\rho}^{b}, \rho \nu_{\rho}^{b^{\prime}}\right), \\
\tau_{\Delta, \Delta^{\prime}}^{>} & =\operatorname{St}\left(\rho \nu_{\rho}^{a}, \rho \nu_{\rho}^{a^{\prime}}\right) \otimes \cdots \otimes \operatorname{St}\left(\rho \nu_{\rho}^{b^{\prime}-a^{\prime}+a}, \rho \nu_{\rho}^{b^{\prime}}\right) \otimes \rho \nu_{\rho}^{b^{\prime}-a^{\prime}+a+1} \otimes \cdots \otimes \rho \nu_{\rho}^{b} .
\end{aligned}
$$

Then the irreducible representation associated to the multisegment $\Delta+\Delta^{\prime}$, denoted $\mathrm{Z}\left(\Delta+\Delta^{\prime}\right)$, is the unique irreducible subquotient $\pi$ of:

$$
\mathrm{Z}(\Delta) \times \mathrm{Z}\left(\Delta^{\prime}\right)
$$

such that one of the following equivalent conditions is fulfilled:

(1) $\tau_{\Delta, \Delta^{\prime}}^{<}$is a subquotient of $\boldsymbol{r}_{\alpha_{\Delta, \Delta^{\prime}}^{<}}(\pi)$;

(2) $\tau_{\Delta, \Delta^{\prime}}^{>}$is a subquotient of $\boldsymbol{r}_{\alpha_{\Delta, \Delta^{\prime}}}(\pi)$;

(3) there are a composition $\alpha$ of $\left(l+l^{\prime}\right) m$ with associated partition $\alpha_{\Delta, \Delta^{\prime}}^{>}$and a residually non-degenerate representation $\tau$ of $\mathrm{M}_{\alpha}$ occurring as a subquotient of $\boldsymbol{r}_{\alpha}(\pi)$. 
The next lemma follows also from the classification theorem of [9].

Lemma 2.11. - Let $\Delta$ and $\Delta^{\prime}$ be two supercuspidal segments such that $\operatorname{deg}(\Delta) \geqslant \operatorname{deg}\left(\Delta^{\prime}\right)$. Let $\pi$ be an irreducible subquotient of $\mathrm{Z}(\Delta) \times \mathrm{Z}\left(\Delta^{\prime}\right)$ different from $\mathrm{Z}\left(\Delta+\Delta^{\prime}\right)$. Let $\mathfrak{m}=\Delta_{1}+\cdots+\Delta_{r}$ be the supercuspidal multisegment attached to $\pi$, and assume that $\Delta_{1}, \ldots, \Delta_{r}$ are ordered so that $\operatorname{deg}\left(\Delta_{1}\right) \geqslant \ldots \geqslant \operatorname{deg}\left(\Delta_{r}\right)$. Then $r \leq 2$ and $\left(\operatorname{deg}\left(\Delta_{1}\right), \operatorname{deg}\left(\Delta_{2}\right)\right) \triangleright\left(\operatorname{deg}(\Delta), \operatorname{deg}\left(\Delta^{\prime}\right)\right)$ (see §1.4).

Proof. - This is a consequence of $[\mathbf{9}, 7.4]$.

\section{Complements on unramified representations of $\mathrm{G}_{m}$}

In this section we prove preliminary results that will be used in the proof of Theorem 0.1. We fix a positive integer $m$.

Lemma 3.1. - Let $\alpha$ be a composition of $m$ and $\rho$ be a smooth representation of the standard Levi subgroup $\mathrm{M}_{\alpha}$. Then $\rho$ is unramified if and only if the representation $\boldsymbol{i}_{\alpha}(\rho)$ is unramified. If this is the case, we have:

$$
\operatorname{dim}_{\mathrm{R}}\left(\rho^{\mathrm{K}_{0} \cap \mathrm{M}_{\alpha}}\right)=\operatorname{dim}_{\mathrm{R}}\left(\boldsymbol{i}_{\alpha}(\rho)^{\mathrm{K}_{0}}\right)=1 .
$$

Proof. - This follows from the isomorphism:

$$
\boldsymbol{i}_{\alpha}(\rho)^{\mathrm{K}_{0}} \simeq \rho^{\mathrm{K}_{0} \cap \mathrm{M}_{\alpha}}
$$

given by $[\mathbf{1 3}$, I.5.6].

The following lemma is a straightforward consequence of the fact that the functor of K-fixed vectors is left exact (see [13, I.4.5]).

Lemma 3.2. - Let $\Pi$ be a smooth representation of $\mathrm{G}_{m}$ and $\mathrm{K}$ be a compact subgroup of $\mathrm{G}_{m}$. Suppose that $\Pi$ does not have any nonzero vector fixed by $\mathrm{K}$. Then no subrepresentation of $\Pi$ has a nonzero vector fixed by $\mathrm{K}$.

Lemma 3.3. - If an irreducible representation is unramified then its cuspidal support is an unramified multisegment (that is, is made of unramified characters of $\mathrm{D}^{\times}$).

Proof. - Let $\pi$ be an unramified representation of $\mathrm{G}_{m}$. Let $\rho_{1}, \ldots, \rho_{r} \in \mathcal{C}_{\mathrm{R}}$ be some cuspidal representations such that $\pi$ is a subrepresentation of:

$$
\rho_{1} \times \cdots \times \rho_{r} .
$$

By Lemma 3.2, we deduce that (3.3) is an unramified representation and then by Lemma 3.1 that for all $1 \leq i \leq r, \rho_{i}$ is an unramified representation. By [8] (see Exemple 2.26 and Remarque 2.27), a cuspidal representation of $\mathrm{G}_{r}$ with $r \geq 2$ does not have any vector fixed by the Iwahori subgroup and hence it is never unramified. This implies that $\rho_{i}$ is unramified if and only if it is an unramified character of $\mathrm{G}_{1}$. The lemma follows.

Another consequence is the following proposition. 
Proposition 3.4. - Let $\mathfrak{m}=\Delta_{1}+\cdots+\Delta_{r}$ be an unramified multisegment such that for all $1 \leq i \neq j \leq r, \Delta_{i}$ and $\Delta_{j}$ are not linked. Then $\mathrm{Z}(\mathfrak{m})$ is an unramified representation.

Proof. - By definition, $\mathrm{Z}(\mathfrak{m})$ is a subquotient of the induced representation:

$$
\mathrm{Z}\left(\Delta_{1}\right) \times \cdots \times \mathrm{Z}\left(\Delta_{r}\right) .
$$

By Proposition 2.10 and our hypothesis, (3.4) is irreducible. We deduce that $\mathrm{Z}(\mathfrak{m})$ is isomorphic to (3.4). By Example 2.9, for all $1 \leq i \leq r$, the representation $\mathrm{Z}\left(\Delta_{i}\right)$ is unramified. Lemma 3.1 implies then that $\mathrm{Z}(\mathfrak{m})$ is unramified.

\section{The case $e=1$}

In this section, we assume that $e=1$, that is, the image of $q^{d}$ in $\mathrm{R}$ is 1 . In this case, the proof of Theorem 0.1 was sketched in $[\mathbf{1 4}, \mathrm{VI} .2]$ for $d=1$.

Let I be the standard Iwahori subgroup of $\mathrm{G}_{m}$ contained in $\mathrm{K}_{0}$. Write $\mathcal{H}\left(\mathrm{G}_{m}, \mathrm{I}\right)$ for the HeckeIwahori algebra made of compactly supported functions from $\mathrm{G}_{m}$ to $\mathrm{R}$ that are bi-invariant under I. This algebra has generators $\mathrm{S}_{1}, \ldots, \mathrm{S}_{m-1}$ and $\mathrm{X}_{1},\left(\mathrm{X}_{1}\right)^{-1}, \ldots, \mathrm{X}_{m},\left(\mathrm{X}_{m}\right)^{-1}$ with relations:

$$
\begin{aligned}
\left(\mathrm{S}_{i}+1\right)\left(\mathrm{S}_{i}-1\right) & =0, \quad i \in\{1, \ldots m-1\}, \\
\mathrm{S}_{i} \mathrm{~S}_{j} & =\mathrm{S}_{j} \mathrm{~S}_{i}, \quad|i-j| \geqslant 2, \\
\mathrm{~S}_{i} \mathrm{~S}_{i+1} \mathrm{~S}_{i} & =\mathrm{S}_{i+1} \mathrm{~S}_{i} \mathrm{~S}_{i+1}, \quad i \in\{1, \ldots m-2\}, \\
\mathrm{X}_{i} \mathrm{X}_{j} & =\mathrm{X}_{j} \mathrm{X}_{i}, \quad i, j \in\{1, \ldots, m\}, \\
\mathrm{X}_{j} \mathrm{~S}_{i} & =\mathrm{S}_{i} \mathrm{X}_{j}, \quad i \notin\{j, j-1\}, \\
\mathrm{S}_{i} \mathrm{X}_{i} \mathrm{~S}_{i} & =\mathrm{X}_{i+1}, \quad i \in\{1, \ldots, m-1\},
\end{aligned}
$$

and $\mathrm{X}_{j}\left(\mathrm{X}_{j}\right)^{-1}=\left(\mathrm{X}_{j}\right)^{-1} \mathrm{X}_{j}=1$ for all $j \in\{1, \ldots, m\}$.

According to Sections 2 and 6 of [15], the map $\pi \mapsto \pi^{\mathrm{I}}$ induces a bijection between irreducible representations of $\mathrm{G}_{m}$ having nonzero I-invariant vectors and simple modules over $\mathcal{H}\left(\mathrm{G}_{m}, \mathrm{I}\right)$.

Proposition 4.1. - The trivial character is the unique unramified irreducible representation of $\mathrm{G}_{m}$ having cuspidal support $1+\cdots+1$ ( $m$ times).

Proof. - Let $\pi$ be an unramified irreducible representation of $\mathrm{G}_{m}$ which embeds in $1 \times \cdots \times 1$. Then $\pi$ has nonzero invariant vectors by I. The space $\pi^{\mathrm{I}}$ is a simple module over $\mathcal{H}\left(\mathrm{G}_{m}, \mathrm{I}\right)$. The generators $\mathrm{X}_{1}, \ldots, \mathrm{X}_{m}$ act as the identity on $\pi^{\mathrm{I}}$, due to the condition on the cuspidal support of $\pi$. Therefore $\pi^{\mathrm{I}}$ can be considered as a simple module over $\mathcal{H}\left(\mathrm{G}_{m}, \mathrm{I}\right) /\left(\mathrm{X}_{1}-1\right)$, that identifies with the spherical Hecke algebra $\mathcal{H}\left(\mathrm{K}_{0}, \mathrm{I}\right)$ generated by $\mathrm{S}_{1}, \ldots, \mathrm{S}_{m-1}$. But $\pi^{\mathrm{K}_{0}}$ is stable under $\mathcal{H}\left(\mathrm{K}_{0}, \mathrm{I}\right)$ and the $\mathrm{S}_{i}$ 's act trivially on it. As $\pi^{\mathrm{I}}$ is simple, we get $\pi^{\mathrm{I}}=\pi^{\mathrm{K}_{0}}$ is the trivial character of $\mathcal{H}\left(\mathrm{K}_{0}, \mathrm{I}\right)$, thus of $\mathcal{H}\left(\mathrm{G}_{m}, \mathrm{I}\right)$. By the bijectivity property of the map $\pi \mapsto \pi^{\mathrm{I}}$, we deduce that $\pi$ is the trivial character of $\mathrm{G}_{m}$.

Proposition 4.2. - Let $\pi$ an unramified irreducible representation of $\mathrm{G}_{m}$. Then there are unramified characters $\chi_{1}, \ldots, \chi_{r}$ of $\mathrm{D}^{\times}$and positive integers $n_{1}, \ldots, n_{r}$ such that $n_{1}+\cdots+n_{r}=m$ and $\pi=\left(\chi_{1} \circ \mathrm{N}_{n_{1}}\right) \times \cdots \times\left(\chi_{r} \circ \mathrm{N}_{n_{r}}\right)$. 
Proof. - Let $\pi$ be an unramified irreducible representation of $\mathrm{G}_{m}$ which embeds in $1 \times \cdots \times 1$. Then $\pi$ has nonzero invariant vectors by $\mathrm{I}$. The space $\pi^{\mathrm{I}}$ is a simple module over $\mathcal{H}\left(\mathrm{G}_{m}, \mathrm{I}\right)$, and it is a submodule of the module $\mathrm{M}$ of I-invariant vectors of $1 \times \cdots \times 1$. Since $e=1$, the generators $\mathrm{X}_{1}, \ldots, \mathrm{X}_{m}$ act as the identity on $\mathrm{M}$, thus on $\pi^{\mathrm{I}}$. Therefore $\pi^{\mathrm{I}}$ can be considered as a simple module over $\mathcal{H}\left(\mathrm{G}_{m}, \mathrm{I}\right) /\left(\mathrm{X}_{1}-1\right)$, that identifies with the spherical Hecke algebra $\mathcal{H}\left(\mathrm{K}_{0}, \mathrm{I}\right)$ generated by $\mathrm{S}_{1}, \ldots, \mathrm{S}_{m-1}$. But $\pi^{\mathrm{K}_{0}}$ is stable under $\mathcal{H}\left(\mathrm{K}_{0}, \mathrm{I}\right)$ and the $\mathrm{S}_{i}$ 's act trivially on it. As $\pi^{\mathrm{I}}$ is simple, we get $\pi^{\mathrm{I}}=\pi^{\mathrm{K}_{0}}$ is the trivial character of $\mathcal{H}\left(\mathrm{K}_{0}, \mathrm{I}\right)$, thus of $\mathcal{H}\left(\mathrm{G}_{m}, \mathrm{I}\right)$. By the bijectivity property of the map $\pi \mapsto \pi^{\mathrm{I}}$, we deduce that $\pi$ is the trivial character of $\mathrm{G}_{m}$.

\section{The case of two segments and $e>1$}

In this section, we assume that $e>1$ and we treat the case where the representation $\pi$ is of the form $\mathrm{Z}\left(\Delta+\Delta^{\prime}\right)$, with $\Delta$ and $\Delta^{\prime}$ some segments.

5.1. The proof of Theorem 0.1 uses the Jacquet functor technique. In the case where $e>1$, we can distinguish irreducible representations by their proper Jacquet functors. First we need to adapt [6, Théorème 5.1] to our situation.

Lemma 5.1. - Let $\pi$ be an irreducible representation of $\mathrm{G}_{n}$ with $n \geq 1$ and $\chi$ be a character of $\mathrm{G}_{1}$. Let $a \geq 1$ be a positive integer. Then the induced representation:

$$
\pi \times \underbrace{\chi \times \chi \times \cdots \times \chi}_{a \text { times }} \quad(\text { resp. } \underbrace{\chi \times \chi \times \cdots \times \chi}_{a \text { times }} \times \pi)
$$

has a unique irreducible subrepresentation.

Proof. - To simplify notation, for every $i \geq 1$, we write:

$$
\chi^{\times i}=\underbrace{\chi \times \chi \times \cdots \times \chi}_{i \text { times }} \text {. }
$$

As we have $e(\chi)=e \geq 2$, Theorem 2.10 implies that $\chi^{\times i}$ is an irreducible representation of $\mathrm{G}_{i}$. Let's prove that $\pi \times \chi^{\times a}$ has a unique irreducible subrepresentation (the proof of the other assertion is similar). First, we reduce to the case where the cuspidal support of $\pi$ is made of elements of $\mathbb{Z}_{\chi}$. Indeed, if this is not the case, then according to [9, Proposition 5.5] we can write $\pi=\pi_{0} \times \pi_{1}$, where the cuspidal support of $\pi_{1}$ is made of elements of $\mathbb{Z}_{\chi}$ and that of $\pi_{0}$ does not contain any element of $\mathbb{Z}_{\chi}$. If $\pi_{1} \times \chi^{\times a}$ has a unique irreducible subrepresentation $\sigma$, then $\pi_{0} \times \sigma$ is the unique irreducible subrepresentation of $\pi \times \chi^{\times a}$. Thus we may and will assume that the cuspidal support of $\pi$ is made of elements of $\mathbb{Z}_{\chi}$.

Let $t$ be the largest integer $i \geq 0$ such that $\pi$ has an irreducible quotient of the form $\tau \times \chi^{\times i}$ for some $\tau \in \operatorname{Irr}\left(\mathrm{G}_{n-i}\right)$.

Lemma 5.2. - Let $t^{\prime}$ be the largest integer $i \geq 0$ such that $\boldsymbol{r}_{(n-i, i)}(\pi)$ possesses an irreducible subquotient of the form $\tau^{\prime} \otimes \chi^{\times i}$ for some $\tau^{\prime} \in \operatorname{Irr}\left(\mathrm{G}_{n-i}\right)$. Then we have $t^{\prime}=t$. 
Proof. - By Frobenius reciprocity, we have $t \leq t^{\prime}$. Given an integer $i \geq 0$, assume that there is an irreducible representation $\tau^{\prime}$ of $\mathrm{G}_{n-i}$ such that $\tau^{\prime} \otimes \chi^{\times i}$ appears as an irreducible subquotient of $\boldsymbol{r}_{(n-i, i)}(\pi)$. Write $\boldsymbol{r}$ for the Jacquet functor $\boldsymbol{r}_{(1, \ldots, 1)}$ and recall that the cuspidal support of $\tau^{\prime}$ is made of elements of $\mathbb{Z}_{\chi}$. If we apply $\boldsymbol{r}$, then we get that $\boldsymbol{r}(\pi)$ has an irreducible subquotient of the form $\varrho=\chi_{1} \otimes \cdots \otimes \chi_{t} \otimes \chi \otimes \cdots \otimes \chi$, where $t=n-i$ and $\chi_{1}, \ldots, \chi_{t} \in \mathbb{Z}_{\chi}$. Since $\boldsymbol{r}(\pi)$ has finite length, it decomposes into a finite direct sum of indecomposable summands. Let $\mathrm{V}$ be an indecomposable summand of $\boldsymbol{r}(\pi)$ that contains $\varrho$ as a subquotient. Since $\varrho$ is a character of the minimal Levi subgroup $\mathrm{M}_{0}=\mathrm{G}_{1} \times \cdots \times \mathrm{G}_{1}$ of $\mathrm{G}_{n}$, it does not have nontrivial extensions with other characters $\varrho^{\prime} \neq \varrho$ of $\mathrm{M}_{0}$. It follows that the irreducible subquotients of $\mathrm{V}$ are all isomorphic to $\varrho$. We thus have a surjective map:

$$
\boldsymbol{r}(\pi) \rightarrow \varrho .
$$

By Frobenius reciprocity, we have a nonzero map from $\boldsymbol{r}_{(n-i, i)}(\pi)$ to $\chi_{1} \times \cdots \times \chi_{t} \otimes \chi^{\times i}$, and thus a surjective map from $\boldsymbol{r}_{(n-i, i)}(\pi)$ to $\tau \otimes \chi^{\times i}$, where $\tau$ is an irreducible subquotient of the product $\chi_{1} \times \cdots \times \chi_{t}$.

Choose $\tau \in \operatorname{Irr}\left(\mathrm{G}_{n-t}\right)$ such that $\operatorname{Hom}_{\mathrm{G}_{n}}\left(\pi, \tau \times \chi^{\times t}\right) \neq 0$. By Lemma 5.2, the representations $\tau$ and $\chi^{\times(t+a)}$ satisfy the conditions of [7, Proposition 2.1]. Thus $\tau \times \chi^{\times(t+a)}$ has a unique irreducible subrepresentation. As $\pi \times \chi^{\times a}$ is a subrepresentation of $\tau \times \chi^{\times(t+a)}$, the lemma follows.

Remark 5.3. - We expect that Lemma 5.1 can be generalized by replacing $\chi$ by any supercuspidal representation $\rho$ of $\mathrm{G}_{r}, r \geq 1$. For this, we need to prove that $\rho$ does not have nontrivial extensions by nonisomorphic unramified twists of $\rho$. Such a question is investigated (in greater generality) in $[\mathbf{1 2}]$. Note that, when $\mathrm{R}$ has positive characteristic, a supercuspidal representation need not be projective mod centre.

5.2. Let $\chi$ be an unramified character of $\mathrm{G}_{1}=\mathrm{D}^{\times}$. Let $\Delta=[a, b]_{\chi}$ and $\Delta^{\prime}=\left[a^{\prime}, b^{\prime}\right]_{\chi}$ be two segments. Write $l$ and $l^{\prime}$ for the lengths of $\Delta$ and $\Delta^{\prime}$ respectively, and suppose that $l \geq l^{\prime}$. The condition $e \geq 2$ means (see Example 2.1) that $e(\chi) \geq 2$. See also that in this case $\nu_{\chi}=\nu^{d}$.

We have the following theorem (see Definition 2.3(2) for the definition of linked segments).

Theorem 5.4. - The segments $\Delta, \Delta^{\prime}$ are linked if and only if $\mathrm{Z}\left(\Delta+\Delta^{\prime}\right)$ is not unramified.

This paragraph is devoted to the proof of this theorem. One implication follows from Proposition 3.4. We prove the other one by induction on the sum $m=l+l^{\prime}$. We suppose henceforth that $\Delta$ and $\Delta^{\prime}$ are linked.

Since the map (2.6) is compatible with unramified twists (see for instance Example 2.9), and since an irreducible representation is unramified if and only if all its unramified twists are, one may assume that $\chi=1$. In this case, $\Delta$ and $\Delta^{\prime}$ are just denoted $[a, b]$ and $\left[a^{\prime}, b^{\prime}\right]$.

Assume that $m=2$ and say $a^{\prime}=a+1$. If $e>2$, the pro-order of $\mathrm{K}_{0}$ is invertible in $\mathrm{R}^{\times}$and hence the functor of $\mathrm{K}_{0}$-invariant vectors is exact [13, I.4.6]. The representation $\nu^{d a} \times \nu^{d a^{\prime}}$ has a unique irreducible unramified subquotient $\mathrm{Z}\left(\left[a, a^{\prime}\right]\right)$, which differs from $\mathrm{Z}\left([a, a]+\left[a^{\prime}, a^{\prime}\right]\right)$. We deduce that the latter representation is not unramified. 
If $e=2$, then the representation $\mathrm{Z}\left([a, a]+\left[a^{\prime}, a^{\prime}\right]\right)$ of $\mathrm{G}_{2}$, by $[\mathbf{1 7}]$, is cuspidal, thus is not unramified.

Suppose that $m \geq 3$ and that the theorem is true for all segments $\Delta, \Delta^{\prime}$ such that $l\left(\Delta+\Delta^{\prime}\right)$ is smaller than $m$. We use the notation of Section 2. Up to equivalence of segments, we distinguish the following cases:

(1) $b=b^{\prime}$ and $\Delta^{-}$and $\Delta^{\prime-}$ are linked.

(2) $b=b^{\prime}$ and $\Delta^{-}$and $\Delta^{\prime-}$ are not linked.

(3) $a=a^{\prime}$ and ${ }^{-} \Delta$ and ${ }^{-} \Delta^{\prime}$ are linked.

(4) $a=a^{\prime}$ and ${ }^{-} \Delta$ and ${ }^{-} \Delta^{\prime}$ are not linked.

(5) $a \not \equiv a^{\prime}(\bmod e)$ and $b \not \equiv b^{\prime}(\bmod e)$.

In each of these cases we will exhibit a representation $V$, which will not be unramified thanks to our inductive argument, and which will have a unique irreducible subrepresentation $\pi$ thanks to Lemma 5.1. We will prove that $\pi$ is isomorphic to $\mathrm{Z}\left(\Delta+\Delta^{\prime}\right)$ : it will follow from Lemma 3.2 that the latter is not unramified. For this, we will prove (see Paragraph 2.3.2) that $\pi$ occurs as a subquotient in $\mathrm{Z}(\Delta) \times \mathrm{Z}\left(\Delta^{\prime}\right)$ and that, for a suitable choice of a composition $\alpha$ of $m$, the Jacquet module $\boldsymbol{r}_{\alpha}(\pi)$ contains a suitable residually non-degenerate irreducible factor.

5.2.1. We start with Case 1. Suppose thus that $b=b^{\prime}$ and $\Delta^{-}, \Delta^{\prime-}$ are linked, and write:

$$
\mathrm{V}=\mathrm{Z}\left(\Delta^{-}+\Delta^{\prime-}\right) \times \nu^{d b} \times \nu^{d b}
$$

The inductive hypothesis together with Lemma 3.1 imply that $\mathrm{V}$ is not unramified. By Lemma $5.1, \mathrm{~V}$ has a unique irreducible subrepresentation $\pi$. We are going to prove that $\pi \simeq \mathrm{Z}\left(\Delta+\Delta^{\prime}\right)$. The theorem will thus follow from Lemma 3.2.

For this (see Paragraph 2.3.2) it is enough to prove that $\pi$ is a subquotient of $\mathrm{Z}(\Delta) \times \mathrm{Z}\left(\Delta^{\prime}\right)$ and that $\tau_{\Delta, \Delta^{\prime}}^{<}$is a subquotient of $\boldsymbol{r}_{\alpha_{\Delta, \Delta^{\prime}}^{<}}(\pi)$. First, by Frobenius reciprocity, we have:

$$
\mathrm{Z}\left(\Delta^{-}+\Delta^{\prime-}\right) \otimes\left(\nu^{d b} \times \nu^{d b}\right) \leq\left[\boldsymbol{r}_{(m-2,2)}(\pi)\right] .
$$

Then, by definition of $\mathrm{Z}\left(\Delta^{-}+\Delta^{\prime-}\right)$ and exactness of the Jacquet functor, we have:

$$
\tau_{\Delta^{-}, \Delta^{\prime}-}^{<} \otimes\left(\nu^{d b} \times \nu^{d b}\right) \leq\left[\boldsymbol{r}_{\alpha_{\Delta, \Delta^{\prime}}^{<}}(\pi)\right] .
$$

But, as $e \geq 2$, we have $\nu^{d b} \times \nu^{d b}=\operatorname{St}\left(\nu^{d b}, \nu^{d b}\right)$ by Paragraph 2.3.1. Thus $\tau_{\Delta, \Delta^{\prime}}^{<}$is a subquotient of $\boldsymbol{r}_{\alpha_{\Delta, \Delta^{\prime}}^{<}}^{<}(\pi)$ as expected. Note that $\mathrm{V}$ is a subrepresentation of:

$$
\mathrm{W}=\mathrm{Z}\left(\Delta^{-}\right) \times \mathrm{Z}\left(\Delta^{\prime-}\right) \times \nu^{d b} \times \nu^{d b} .
$$

The representation $\tau_{\Delta, \Delta^{\prime}}^{<}$occurs with multiplicity 1 in $\boldsymbol{r}_{\alpha_{\Delta, \Delta^{\prime}}^{<}}\left(\mathrm{Z}(\Delta) \times \mathrm{Z}\left(\Delta^{\prime}\right)\right)$. By the geometric lemma, and thanks to the fact that $e \geq 2$, it also occurs in $\boldsymbol{r}_{\alpha_{\Delta, \Delta^{\prime}}^{<}}(\mathrm{W})$ with multiplicity 1 . If $\pi$ is not a subquotient of $\mathrm{Z}(\Delta) \times \mathrm{Z}\left(\Delta^{\prime}\right)$, then:

$$
[\pi]+\left[\mathrm{Z}(\Delta) \times \mathrm{Z}\left(\Delta^{\prime}\right)\right] \leq[\mathrm{W}]
$$

Thus, by exactness of the Jacquet functor, $\tau_{\Delta, \Delta^{\prime}}^{<}$occurs in $\boldsymbol{r}_{\alpha_{\Delta, \Delta^{\prime}}^{<}}(\mathrm{W})$ with multiplicity 2: contradiction. 
5.2.2. We now treat Case 2. Suppose that $b=b^{\prime}$ and $\Delta^{-}, \Delta^{\prime-}$ are not linked. Remark that we have $a \leqslant a^{\prime} \leqslant b$.

Lemma 5.5. - We have $l^{\prime} \leqslant e$ and e divides $l$.

Proof. - According to Corollary 2.6, there is a $c^{\prime} \in \mathbb{Z}$ such that $a^{\prime} \leqslant c^{\prime} \leqslant b$ and $c^{\prime}$ is congruent to $b+1$ or $a-1 \bmod e$, and there is no $d^{\prime} \in \mathbb{Z}$ such that $a^{\prime} \leqslant d^{\prime} \leqslant b-1$ and $d^{\prime}$ is congruent to $b$ or $a-1 \bmod e$. The second condition implies that $b-a^{\prime}<e$, or equivalently $l^{\prime} \leqslant e$.

Assume that $a^{\prime} \leqslant c^{\prime}<b$. Then $c^{\prime} \equiv b+1(\bmod e)$ which contradicts the fact that $l^{\prime} \leqslant e$. It follows that $c^{\prime}=b$. Thus $b \equiv a-1(\bmod e)$, or equivalently $e$ divides $l$.

In other words, we have $b-e+1 \leq a^{\prime} \leq b$ and there is a $t \geq 1$ such that $a=b-t e+1$. We now distinguish two cases: $l^{\prime} \geqslant 2$ and $l^{\prime}=1$.

5.2.2.1. Let us first assume that $a^{\prime} \neq b$, that is $l^{\prime} \geqslant 2$, and write:

$$
\mathrm{V}=\nu^{d a^{\prime}} \times \mathrm{Z}\left({ }^{-} \Delta^{\prime}+\Delta\right) .
$$

It has a unique irreducible subrepresentation $\pi$ and, since ${ }^{-} \Delta^{\prime}, \Delta$ are linked, it is not unramified. We will prove that $\pi$ is isomorphic to $\mathrm{Z}\left(\Delta+\Delta^{\prime}\right)$. For this, we will show that there is a composition $\alpha$ of $m$, with associated partition $\alpha_{\Delta, \Delta^{\prime}}^{>}$, and a residually non-degenerate representation $\tau$ of $\mathrm{M}_{\alpha}$ occurring as a subquotient of $\boldsymbol{r}_{\alpha}(\pi)$. Then we will prove that $\pi$ occurs as a subquotient of $\mathrm{Z}(\Delta) \times \mathrm{Z}\left(\Delta^{\prime}\right)$. By Frobenius reciprocity, we have:

$$
\nu^{d a^{\prime}} \otimes \mathrm{Z}\left({ }^{-} \Delta^{\prime}+\Delta\right) \leq\left[\boldsymbol{r}_{(1, m-1)}(\pi)\right] .
$$

By definition of $\mathrm{Z}\left({ }^{-} \Delta^{\prime}+\Delta\right)$ and exactness of the Jacquet functor, $\nu^{d a^{\prime}} \otimes \tau_{\Delta,-\Delta^{\prime}}^{<}$occurs as a subquotient of $\boldsymbol{r}_{\alpha}(\pi)$, where $\alpha$ is the composition:

$$
\alpha=(\underbrace{1,1, \ldots, 1}_{a^{\prime}-a+2}, \underbrace{2,2, \ldots, 2}_{b-a^{\prime}}) .
$$

Thus the irreducible representation:

$$
\nu^{d a^{\prime}} \otimes \nu^{d a} \otimes \nu^{d(a+1)} \otimes \cdots \otimes \nu^{d a^{\prime}} \otimes \operatorname{St}\left(\nu^{d\left(a^{\prime}+1\right)}, \nu^{d\left(a^{\prime}+1\right)}\right) \otimes \cdots \otimes \operatorname{St}\left(\nu^{d b}, \nu^{d b}\right)
$$

denoted $\tau$, occurs as subquotient of $\boldsymbol{r}_{\alpha}(\pi)$. Write $\alpha^{0}$ for the composition of $m$ defined by:

$$
\alpha^{0}=(2, \underbrace{1,1, \ldots, 1}_{a^{\prime}-a}, \underbrace{2,2, \ldots, 2}_{b-a^{\prime}}) \text {. }
$$

Then there is an irreducible representation $\rho$ of $\mathrm{G}_{2}$ such that $\nu^{d a^{\prime}} \otimes \nu^{d a}$ occurs as a subquotient of $\boldsymbol{r}_{(1,1)}(\rho)$ and:

$$
\rho \otimes \nu^{d(a+1)} \otimes \cdots \otimes \nu^{d a^{\prime}} \otimes \operatorname{St}\left(\nu^{d\left(a^{\prime}+1\right)}, \nu^{d\left(a^{\prime}+1\right)}\right) \otimes \cdots \otimes \operatorname{St}\left(\nu^{d b}, \nu^{d b}\right)
$$

denoted $\tau_{0}$ occurs as a subquotient of $\boldsymbol{r}_{\alpha^{0}}(\pi)$. Since $a^{\prime} \neq b$ and by Paragraph 2.3.1, $\rho$ is isomorphic to $\operatorname{St}\left(\nu^{d a^{\prime}}, \nu^{d a}\right)$. This proves our first assertion. We prove now that $\pi$ is a subquotient of $\mathrm{Z}(\Delta) \times \mathrm{Z}\left(\Delta^{\prime}\right)$. We write $\mathrm{W}=\nu^{d a^{\prime}} \times \mathrm{Z}(\Delta) \times \mathrm{Z}\left({ }^{-} \Delta^{\prime}\right)$. 
Suppose that $a^{\prime} \neq a$. Then $\tau$ appears with multiplicity 1 in $\boldsymbol{r}_{\alpha}\left(\mathrm{Z}(\Delta) \times \mathrm{Z}\left(\Delta^{\prime}\right)\right)$ and $\boldsymbol{r}_{\alpha}(\mathrm{W})$. Since $\mathrm{V}$ is a subquotient of $\mathrm{W}$, we have that $\pi$ is a subquotient of $\mathrm{W}$. Hence, if $\pi$ is not a subquotient of $\mathrm{Z}(\Delta) \times \mathrm{Z}\left(\Delta^{\prime}\right)$, then:

$$
[\pi]+\left[\mathrm{Z}(\Delta) \times \mathrm{Z}\left(\Delta^{\prime}\right)\right] \leq[\mathrm{W}]
$$

By exactness of the Jacquet functor, $\tau$ appears in $\boldsymbol{r}_{\alpha}(\mathrm{W})$ with multiplicity 2: contradiction.

Suppose that $a^{\prime}=a$, thus $t=1$ and $\Delta^{\prime}=\Delta$. We write:

$$
\mathrm{X}=\nu^{d a} \times \nu^{d a} \times \mathrm{Z}\left({ }^{-} \Delta\right) \times \mathrm{Z}\left({ }^{-} \Delta^{\prime}\right) .
$$

The representation $\tau_{0}$ appears with multiplicity 1 in $\boldsymbol{r}_{\alpha^{0}}\left(\mathrm{Z}(\Delta) \times \mathrm{Z}\left(\Delta^{\prime}\right)\right)$ and $\boldsymbol{r}_{\alpha^{0}}(\mathrm{X})$. Since $\mathrm{V}$ is a subquotient of $\mathrm{X}$, we have that $\pi$ is a subquotient of $\mathrm{X}$. Hence, if $\pi$ is not a subquotient of $\mathrm{Z}(\Delta) \times \mathrm{Z}\left(\Delta^{\prime}\right)$, then:

$$
[\pi]+\left[\mathrm{Z}(\Delta) \times \mathrm{Z}\left(\Delta^{\prime}\right)\right] \leq[\mathrm{X}]
$$

By exactness of the Jacquet functor, $\tau_{0}$ appears in $\boldsymbol{r}_{\alpha^{0}}(\mathrm{X})$ with multiplicity 2: contradiction.

5.2.2.2. Suppose now that $a^{\prime}=b$, that is $\Delta^{\prime}=[b, b]$ has length 1 . This case is particular and we treat it by a different method. We write $\mathrm{V}=\mathrm{Z}([a, b]) \times \nu^{d b}$ and $\pi=\mathrm{Z}([a, b]+[b, b])$. Write $\mathrm{K}_{1}$ for the normal subgroup of $\mathrm{K}_{0}$ made of those matrices that are congruent to the identity mod the maximal ideal of $\mathcal{O}_{\mathrm{D}}$. We identify the quotient $\mathrm{K}_{0} / \mathrm{K}_{1}$ with the general linear group $\mathrm{GL}_{m}(k)$ where $k$ is the residue field of $\mathrm{D}$, which has cardinality $q^{d}$. Thus the space of $\mathrm{K}_{1}$-fixed vectors of any smooth representation of $\mathrm{G}$ is naturally endowed with an action of $\mathrm{GL}_{m}(k)$. Note that $m=l+1$. Write $\mathrm{X}$ for the projective space of $k^{m}$.

Lemma 5.6. - $\mathrm{V}^{\mathrm{K}_{1}}$ identifies with the representation of $\mathrm{GL}_{m}(k)$ on the space of functions from $\mathrm{X}$ to $\mathrm{R}$.

Proof. - Let $\mathrm{P}$ be the standard parabolic subgroup $\mathrm{P}_{(l, 1)}$ of $\mathrm{G}$. Restricting functions from $\mathrm{G}$ to $\mathrm{K}_{0}$ induces a bijective and $\mathrm{K}_{0}$-equivariant map from $\mathrm{V}^{\mathrm{K}_{1}}$ to the representation of $\mathrm{K}_{0}$ induced from the trivial character of $\mathrm{P} \cap \mathrm{K}_{0}$. The latter representation identifies with the representation of $\mathrm{GL}_{m}(k)$ on the space of functions from $\mathrm{X}$ to $\mathrm{R}$, since $\left(\mathrm{P} \cap \mathrm{K}_{0}\right) \backslash \mathrm{K}_{0}$ identifies with $\mathrm{X}$.

Lemma 5.7. - The representation $\mathrm{V}^{\mathrm{K}_{1}}$ is semisimple of length 2 , and the trivial character $1_{m}$ of $\mathrm{GL}_{m}(k)$ occurs with multiplicity 1 in it.

Proof. - We identify $\mathrm{V}^{\mathrm{K}_{1}}$ with the space $\mathrm{S}$ of functions from $\mathrm{X}$ to $\mathrm{R}$, which is the representation of $\mathrm{GL}_{m}(k)$ parabolically induced from the trivial character of the standard Levi subgroup of $\mathrm{GL}_{m}(k)$ associated with the composition $(l, 1)$.

According to James's classification [5] (see also [9, §3.3]), $\mathrm{S}$ is made of a nontrivial irreducible subquotient $\mathrm{S}_{0}$, occuring with multiplicity 1 , and of the trivial character $1_{m}$, occuring with some multiplicity $n \geqslant 1$. Given $f \in \mathrm{S}$, write $\psi(f)$ for the sum of the values of $f$ in $\mathrm{R}$. Then $\psi$ is a homomorphism from $\mathrm{S}$ to the trivial character $1_{m}$.

Lemma 5.8. - The trivial character $1_{m}$ does not occur as a subrepresentation of $\operatorname{Ker}(\psi)$. 
Proof. - Assume that $1_{m}$ occurs as a subrepresentation of $\operatorname{Ker}(\psi)$, and write $f_{1}$ for the constant function equal to 1 . Then $\psi\left(f_{1}\right)$ is equal to the cardinality of $\mathrm{X}$, thus we have:

$$
0=\psi\left(f_{1}\right)=\frac{q^{m d}-1}{q^{d}-1}
$$

in R. It follows that $q^{m d}$ is congruent to $1 \bmod \ell$, that is $e$ divides $m=l+1$. The contradiction follows from Lemma 5.5.

It follows that the socle of $\operatorname{Ker}(\psi)$ is equal to $\mathrm{S}_{0}$. Since $\mathrm{S}$ is selfcontragredient, $\mathrm{S}_{0}$ is selfcontragredient too, $1_{m}$ occurs as a subrepresentation of $\mathrm{S}$ and $\mathrm{S}_{0}$ as a quotient of $\mathrm{S}$. Thus $1_{m}$ and $\mathrm{S}_{0}$ are direct summands in $\mathrm{S}$, that is we have $\mathrm{S}=\mathrm{S}_{0} \oplus \mathrm{S}^{\prime}$ for some subrepresentation $\mathrm{S}^{\prime}$ of $\mathrm{S}$ which is made of $1_{m}$ with multiplicity $n$, and $1_{m}$ is a direct summand in $\mathrm{S}^{\prime}$. This gives us:

$$
\operatorname{Ker}(\psi)=\mathrm{S}_{0} \oplus\left(\operatorname{Ker}(\psi) \cap \mathrm{S}^{\prime}\right) .
$$

If $\operatorname{Ker}(\psi)$ is not reduced to $\mathrm{S}_{0}$, then $1_{m}$ occurs as a subrepresentation of $\operatorname{Ker}(\psi)$, which contradicts Lemma 5.8.

We thus have:

$$
V^{\mathrm{K}_{1}}=\mathrm{Z}([a-1, b])^{\mathrm{K}_{1}} \oplus \pi^{\mathrm{K}_{1}}=1_{m} \oplus \pi^{\mathrm{K}_{1}}=1_{m} \oplus \mathrm{S}_{0} .
$$

It follows that $\pi^{\mathrm{K}_{1}}=\mathrm{S}_{0}$ is irreducible nontrivial, thus $\pi^{\mathrm{K}_{0}}$ is zero and $\pi$ is not unramified.

5.2.3. The proof in Case 3 is similar to that of Case 1, using $\tau_{\Delta, \Delta^{\prime}}^{>}$instead of $\tau_{\Delta, \Delta^{\prime}}^{<}$

5.2.4. The proof in Case 4 is similar to that of Case 2. Note that $a \leq b^{\prime} \leq a+e-1$ and there is an integer $t \geq 1$ such that $b=a+t e-1$.

5.2.5. We now treat Case 5. This case itself decomposes into seven subcases:

(1) $l>l^{\prime}$ and $\Delta$ precedes $\Delta^{\prime}$.

(2) $l>l^{\prime}$ and $\Delta^{\prime}$ precedes $\Delta$.

(3) $l=l^{\prime}$ and $a \not \equiv a^{\prime}-1(\bmod e)$ and ${ }^{-} \Delta, \Delta^{\prime}$ are linked.

(4) $l=l^{\prime}$ and $a^{\prime} \not \equiv a-1(\bmod e)$ and ${ }^{-} \Delta^{\prime}, \Delta$ are linked.

(5) $l=l^{\prime}$ and $b \not \equiv b^{\prime}+1(\bmod e)$ and $\Delta^{-}, \Delta^{\prime}$ are linked.

(6) $l=l^{\prime}$ and $b^{\prime} \not \equiv b+1(\bmod e)$ and $\Delta^{-}, \Delta^{\prime}$ are linked.

(7) $l=l^{\prime}$ and $e=2$.

Note that Subcases 4 and 6 follow respectively from 3 and 5 by exchanging $\Delta$ and $\Delta^{\prime}$ and will not be treated.

We write $\mathrm{V}=\nu^{d a} \times \mathrm{Z}\left({ }^{-} \Delta+\Delta^{\prime}\right)$ and $\mathrm{W}=\nu^{d a} \times \mathrm{Z}\left({ }^{-} \Delta\right) \times \mathrm{Z}\left(\Delta^{\prime}\right)$ and $\pi$ for the unique irreducible subrepresentation of V. By Frobenius reciprocity, $\nu^{d a} \otimes \mathrm{Z}\left({ }^{-} \Delta+\Delta^{\prime}\right)$ occurs as a subquotient of the Jacquet module $\boldsymbol{r}_{(1, m-1)}(\pi)$.

Subcase 1: In this first subcase, ${ }^{-} \Delta$ precedes $\Delta^{\prime}$. By definition of $\mathrm{Z}\left({ }^{-} \Delta+\Delta^{\prime}\right)$ and exactness of the Jacquet functor, we have:

$$
\nu^{d a} \otimes \tau_{-\Delta, \Delta^{\prime}}^{<} \leq\left[\boldsymbol{r}_{\alpha_{\Delta, \Delta^{\prime}}^{<}}(\pi)\right]
$$


Hence $\tau_{\Delta, \Delta^{\prime}}^{<}$occurs in $\boldsymbol{r}_{\alpha_{\Delta, \Delta^{\prime}}^{<}}(\pi)$ with multiplicity 1 . Thanks to the fact that $a \not \equiv a^{\prime}(\bmod e)$, it also occurs with multiplicity 1 in $\boldsymbol{r}_{\alpha_{\Delta, \Delta^{\prime}}^{<}}(\mathrm{W})$ and $\boldsymbol{r}_{\alpha_{\Delta, \Delta^{\prime}}^{<}}\left(\mathrm{Z}(\Delta) \times \mathrm{Z}\left(\Delta^{\prime}\right)\right)$. If $\pi$ is not a subquotient of $\mathrm{Z}(\Delta) \times \mathrm{Z}\left(\Delta^{\prime}\right)$ then:

$$
[\pi]+\left[\mathrm{Z}(\Delta) \times \mathrm{Z}\left(\Delta^{\prime}\right)\right] \leq[\mathrm{W}]
$$

By exactness of the Jacquet functor, $\tau_{\Delta, \Delta^{\prime}}^{<}$appears in $\boldsymbol{r}_{\alpha_{\Delta, \Delta^{\prime}}^{<}}(\mathrm{W})$ with multiplicity 2: contradiction.

Subcase 2: The proof is the same as in the previous case, using now $\tau_{\Delta, \Delta^{\prime}}^{>}$instead of $\tau_{\Delta, \Delta^{\prime}}^{<}$.

Subcase 3: Suppose that $l=l^{\prime}$ and $a \neq \equiv a^{\prime}-1(\bmod e)$ and ${ }^{-} \Delta, \Delta^{\prime}$ are linked. We have:

$$
\nu^{d a} \otimes \tau_{-\Delta, \Delta^{\prime}}^{<} \leq\left[\boldsymbol{r}_{\alpha^{0}}(\pi)\right],
$$

where $\alpha^{0}$ is the composition of $2 l$ defined by:

$$
\alpha^{0}=(1,1, \underbrace{2,2, \ldots, 2}_{l-1}) .
$$

Thus, by definition of $\tau_{\Delta, \Delta^{\prime}}^{<}$, we have:

$$
\nu^{d a} \otimes \nu^{d a^{\prime}} \otimes \operatorname{St}\left(\nu^{d(a+1)}, \nu^{d\left(a^{\prime}+1\right)}\right) \otimes \cdots \otimes \operatorname{St}\left(\nu^{d b}, \nu^{d b^{\prime}}\right) \leq\left[\boldsymbol{r}_{\alpha^{0}}(\pi)\right] .
$$

Then there is an irreducible representation $\rho$ of $\mathrm{G}_{2}$ such that $\nu^{d a} \otimes \nu^{d a^{\prime}} \leq\left[\boldsymbol{r}_{(1,1)}(\rho)\right]$ and:

$$
\tau=\rho \otimes \operatorname{St}\left(\nu^{d(a+1)}, \nu^{d\left(a^{\prime}+1\right)}\right) \otimes \cdots \otimes \operatorname{St}\left(\nu^{d b}, \nu^{d b^{\prime}}\right)
$$

occurs as a subquotient of $\boldsymbol{r}_{\alpha^{0}}(\pi)$. By the classification of irreducible representations of $\mathrm{G}_{2}$ (see Paragraph 2.3.1) and the assumption $a \not \equiv a^{\prime}-1(\bmod e)$, we have $\rho=\operatorname{St}\left(\nu^{d a}, \nu^{d a^{\prime}}\right)$. Thus $\tau_{\Delta, \Delta^{\prime}}^{<}$ occurs as a subquotient of $\boldsymbol{r}_{\alpha_{\Delta, \Delta^{\prime}}^{<}}(\pi)$. Thanks to the fact that $a \not \equiv a^{\prime}(\bmod e)$, it occurs also with multiplicity 1 in $\left[\boldsymbol{r}_{\alpha_{\Delta, \Delta^{\prime}}^{<}}(\mathrm{W})\right]$ and in $\left[\boldsymbol{r}_{\alpha_{\Delta, \Delta^{\prime}}^{<}}\left(\mathrm{Z}(\Delta) \times \mathrm{Z}\left(\Delta^{\prime}\right)\right)\right]$. If $\pi$ is not a subquotient of $\mathrm{Z}(\Delta) \times \mathrm{Z}\left(\Delta^{\prime}\right)$ then:

$$
[\pi]+\left[\mathrm{Z}(\Delta) \times \mathrm{Z}\left(\Delta^{\prime}\right)\right] \leq[\mathrm{W}] .
$$

By exactness of the Jacquet functor, $\tau_{\Delta, \Delta^{\prime}}^{<}$appears in $\boldsymbol{r}_{\alpha_{\Delta, \Delta^{\prime}}^{<}}(\mathrm{W})$ with multiplicity 2: contradiction.

Subcase 5: The proof of Subcase 5 is the same as in Subcase 3, using now $\tau_{\Delta, \Delta^{\prime}}^{>}$instead of $\tau_{\Delta, \Delta^{\prime}}^{<}$

Subcase 7: Suppose that $l=l^{\prime}$ and $e=2$. This is the easiest subcase. By [9, Théorème $7.32(3)]$, the cuspidal support of $\mathrm{Z}\left(\Delta+\Delta^{\prime}\right)$ is not made of unramified characters so, by Lemma 3.3, $\mathrm{Z}\left(\Delta+\Delta^{\prime}\right)$ cannot be an unramified representation!

\section{The general case}

In this section we prove the main theorem of this article.

Theorem 6.1. - Let $\mathfrak{m}=\Delta_{1}+\cdots+\Delta_{r}$ be a multisegment and write $\pi=\mathrm{Z}(\mathfrak{m})$. Then $\pi$ is unramified if and only if $\mathfrak{m}$ is unramified and, for all $1 \leq i<j \leq r$, the segments $\Delta_{i}$ and $\Delta_{j}$ are not linked. 
Proof. - By Proposition 3.4, the condition on $\mathfrak{m}$ is sufficient. Suppose that $\pi$ is unramified. By Lemma 3.3, the multisegment $\mathfrak{m}$ is unramified.

If $e=1$, the theorem follows from Proposition 4.2. Suppose $e>1$ and let $I$ be the set of sequences $\left(\Delta_{1}^{\prime}, \ldots, \Delta_{r}^{\prime}\right)$ of segments such that $\pi$ is a subrepresentation of $\mathrm{Z}\left(\Delta_{1}^{\prime}\right) \times \cdots \times \mathrm{Z}\left(\Delta_{r}^{\prime}\right)$. Let $\left(\Delta_{1}^{\prime}, \ldots, \Delta_{r}^{\prime}\right) \in I$ be so that the partition associated to the composition $\left(l\left(\Delta_{1}^{\prime}\right), \ldots, l\left(\Delta_{r}^{\prime}\right)\right)$ is minimal. Suppose that there exist $1 \leq i<j \leq r$, such that $\Delta_{i}^{\prime}$ and $\Delta_{j}^{\prime}$ are linked. By Theorem 2.10 , we can suppose that $j=i+1$. Then, by Lemma 2.11 , every subquotient of $\mathrm{Z}\left(\Delta_{i}^{\prime}\right) \times \mathrm{Z}\left(\Delta_{j}^{\prime}\right)$ is of the form $\mathrm{Z}\left(\delta+\delta^{\prime}\right)$, where $\delta+\delta^{\prime}$ is a multisegment such that:

- either $\delta+\delta^{\prime}=\Delta_{i}^{\prime}+\Delta_{i+1}^{\prime}$, or

- the partition associated to the pair $\left(l(\delta), l\left(\delta^{\prime}\right)\right)$ is bigger than the partition associated to the pair $\left(l\left(\Delta_{i}^{\prime}\right), l\left(\Delta_{i+1}^{\prime}\right)\right)$.

We deduce that $\pi$ is a subrepresentation of:

$$
\mathrm{Z}\left(\Delta_{1}^{\prime}\right) \times \cdots \times \mathrm{Z}\left(\Delta_{i-1}^{\prime}\right) \times \mathrm{Z}\left(\delta+\delta^{\prime}\right) \times \mathrm{Z}\left(\Delta_{i+2}\right) \times \cdots \times \mathrm{Z}\left(\Delta_{r}^{\prime}\right) .
$$

with $\delta+\delta^{\prime}$ as above.

If $\delta$ and $\delta^{\prime}$ are linked (in particular in the first case), then Theorem 5.4 implies that $\mathrm{Z}\left(\delta+\delta^{\prime}\right)$ is not unramified. By Lemma 3.1, the representation (6.1) is not unramified. By Lemma 3.2 we deduce that $\pi$ is not unramified, which contradicts our hypothesis.

If $\delta$ and $\delta^{\prime}$ are not linked (and thus we are in the second case), then Theorem 2.10 implies that $\mathrm{Z}\left(\delta+\delta^{\prime}\right)$ is isomorphic to $\mathrm{Z}(\delta) \times \mathrm{Z}\left(\delta^{\prime}\right)$, hence $\pi$ is a subrepresentation of:

$$
\mathrm{Z}\left(\Delta_{1}^{\prime}\right) \times \cdots \times \mathrm{Z}\left(\Delta_{i-1}^{\prime}\right) \times \mathrm{Z}(\delta) \times \mathrm{Z}\left(\delta^{\prime}\right) \times \mathrm{Z}\left(\Delta_{i+2}\right) \times \cdots \times \mathrm{Z}\left(\Delta_{r}^{\prime}\right) .
$$

This contradicts, by Lemma 1.1, the minimality of the partition associated to $\left(\Delta_{1}^{\prime}, \ldots, \Delta_{r}^{\prime}\right)$.

Thus for all $1 \leq i<j \leq r, \Delta_{i}^{\prime}$ and $\Delta_{j}^{\prime}$ are not linked. We deduce that $\pi=\mathrm{Z}\left(\Delta_{1}^{\prime}+\cdots+\Delta_{r}^{\prime}\right)$ and this finishes the proof of the theorem.

Let $\ell$ be a prime number different from $p$. We denote by $\mathbb{Q}_{\ell}$ the field of $\ell$-adic numbers and $\mathbb{F}_{\ell}$ its residue field. We fix an algebraic closure $\overline{\mathbb{Q}}_{\ell}$ of $\mathbb{Q}_{\ell}$ and we denote by $\overline{\mathbb{F}}_{\ell}$ its residue field, which is an algebraic closure of $\mathbb{F}_{\ell}$.

We say that an irreducible $\overline{\mathbb{F}}_{\ell}$-representation $\pi$ of $\mathrm{G}_{m}, m \geqslant 1$, can be lifted to $\overline{\mathbb{Q}}_{\ell}$ if there exists an integral $\overline{\mathbb{Q}}_{\ell}$-representation $\tilde{\pi}$ such that $\pi$ is the reduction modulo $\ell$ of $\tilde{\pi}$ (see [9]).

Corollary 6.2. - An irreducible representation $\pi$ of $\mathrm{G}_{m}, m \geqslant 1$, is unramified if and only if it is irreducibly induced from an unramified character of a parabolic subgroup. In this case we have that $\operatorname{dim}_{\mathrm{R}}\left(\pi^{\mathrm{K}_{0}}\right)=1$. Every irreducible unramified $\overline{\mathbb{F}}_{\ell}$-representation can be lifted to $\overline{\mathbb{Q}}_{\ell}$.

This corollary was conjectured in [14, VI.3].

Proof. - The first part of the corollary follows from the fact that isomorphism classes of unramified characters of $\mathrm{G}_{m}$ are in bijection with classes of unramified segments of length $m$. The identity (3.2) implies that $\operatorname{dim}\left(\pi^{\mathrm{K}_{0}}\right)=1$. Every $\overline{\mathbb{F}}_{\ell}$-character can be lifted to an integral $\overline{\mathbb{Q}}_{\ell}$-character. The last part of the corollary follows then from $[\mathbf{9}, 1.2 .3]$. 


\section{References}

[1] J. Bernstein \& A. Zelevinski - "Induced representations of reductive p-adic groups I", Ann. Sci. École Norm. Sup. (4) 10 (1977), p. 441-472.

[2] A. BorEL - "Admissible representations of a semi-simple group over a local field with vector fixed under an Iwahori subgroup", Inv. Math 35 (1976), pp.233-259.

[3] P. CARTIER - "Representations of p-adic groups: a survey", Automorphic forms, representations and L-functions (Proc. Sympos. Pure Math., Oregon State Univ., Corvallis, Ore., 1977), Part 1. Proc. Sympos. Pure Math., XXXIII. p. 111-155. Amer. Math. Soc. Providence, R.I. (1979).

[4] L. Clozel, M. Harris \& R. TAYlor - "Automorphy for some $\ell$-adic lifts of automorphic mod $\ell$ Galois representations Publ. Math. I.H.E.S. 108, (2008), pp. 1-181.

[5] G. JAMES - "The irreducible representations of the finite general linear groups", Proc. London Math. Soc. (3) 52 (1986), no. 2, p. 236-268.

[6] A. Mínguez - "Sur l'irréductibilité d'une induite parabolique", J. Reine Angew. Math. 629 (2009), p. $107-131$.

[7] A. Mínguez \& V. SÉcherre - "Représentations banales de GL $m$ (D)". To appear in Compositio Math.

[8] _ "Types modulo $\ell$ pour les formes intérieures de $\mathrm{GL}_{n}$ sur un corps local non archimédien", preprint. Available at http://www.math.jussieu.fr/ minguez/, 2012.

[9] _ "Représentations lisses modulo $\ell$ de $\mathrm{GL}_{m}(\mathrm{D})$ ", preprint. Available at http://www.math.jussieu.fr/ minguez/, 2012.

[10] I. SATAKE - "Theory of spherical functions on reductive algebraic groups over p-adic fields", Publ. Math. I.H.E.S. 18, (1963) p. 5-69.

[11] M. TADić - "Spherical unitary dual of general linear group over non-archimedean local field, Ann. Inst. Fourier, Grenoble 36, 2 (1986), p. 47-55.

[12] V. SÉcherre \& S. Stevens - Blocks modulo l for smooth representations of $\mathrm{GL}_{m}(\mathrm{D})$, in progress.

[13] M.-F. VignÉRAs - Représentations l-modulaires d'un groupe réductif $p$-adique avec $l \neq p$, Progress in Mathematics, vol. 137, Birkhäuser Boston Inc., Boston, MA, 1996.

[14] _ "Induced R-representations of $p$-adic reductive groups", Selecta Math. (N.S.) 4 (1998), no. 4, p. 549-623. With an appendix by Alberto Arabia.

[15] _ "Irreducible modular representations of a reductive $p$-adic group and simple modules for Hecke algebras", in European Congress of Mathematics, Vol. I (Barcelona, 2000), Progr. Math., vol. 201, Birkhäuser, Basel, 2001, p. 117-133.

[16] _ "Correspondance de Langlands semi-simple pour $\mathrm{GL}_{n}(\mathrm{~F})$ modulo $\ell \neq p$ ", Invent. Math 144 (2001), p. 177-223.

[17] _ "Représentations de $\mathrm{GL}(2, F)$ en caractéristique $\ell, F$ corps $p$-adique, $p \neq \ell$ ", Compos. Math. 72, 1989, 33-66.

Alberto Mínguez, Institut de Mathématiques de Jussieu, Université Paris 6, 4 place Jussieu, 75005, Paris, France. URL: http://www.math.jussieu.fr/ minguez/ • E-mail : minguez@math.jussieu.fr

Vincent SÉcherRe, Université de Versailles Saint-Quentin-en-Yvelines, Laboratoire de Mathématiques de Versailles, 45 avenue des Etats-Unis, 78035 Versailles cedex, France

E-mail : vincent.secherre@math.uvsq.fr 